\title{
Low-dimensional models for turbulent plane Couette flow in a minimal flow unit
}

\author{
By T. R. SMITH ${ }^{1} \dagger$, J. MOEHLIS ${ }^{2} \ddagger$ AND P. HOLMES $S^{1,2}$ \\ ${ }^{1}$ Department of Mechanical and Aerospace Engineering, Princeton University, \\ Princeton, NJ 08544-5263, USA \\ ${ }^{2}$ Program in Applied and Computational Mathematics, Princeton University, \\ Princeton, NJ 08544-1000, USA
}

(Received 19 December 2003 and in revised form 7 March 2005)

We model turbulent plane Couette flow in the minimal flow unit (MFU) - a domain whose spanwise and streamwise extent is just sufficient to maintain turbulence by expanding the velocity field as a sum of optimal modes calculated via proper orthogonal decomposition from numerical data. Ordinary differential equations are obtained by Galerkin projection of the Navier-Stokes equations onto these modes. We first consider a 6-mode (11-dimensional) model and study the effects of including losses to neglected modes. Ignoring these, the model reproduces turbulent statistics acceptably, but fails to reproduce dynamics; including them, we find a stable periodic orbit that captures the regeneration cycle dynamics and agrees well with direct numerical simulations. However, restriction to as few as six modes artificially constrains the relative magnitudes of streamwise vortices and streaks and so cannot reproduce stability of the laminar state or properly account for bifurcations to turbulence as Reynolds number increases. To address this issue, we develop a second class of models based on 'uncoupled' eigenfunctions that allow independence among streamwise and cross-stream velocity components. A 9-mode (31-dimensional) model produces bifurcation diagrams for steady and periodic states in qualitative agreement with numerical Navier-Stokes solutions, while preserving the regeneration cycle dynamics. Together, the models provide empirical evidence that the 'backbone' for MFU turbulence is a periodic orbit, and support the roll-streak-breakdown-roll reformation picture of shear-driven turbulence.

\section{Introduction}

In this paper we continue a study of low-dimensional models of plane Couette flow begun in Moehlis et al. (2002). That paper and the present one take up earlier work begun by Aubry et al. (1988) and Sanghi \& Aubry (1993), cf. Berkooz, Holmes \& Lumley (1991), Berkooz et al. (1994), Podvin \& Lumley (1998), Podvin (2001), and Sirovich (1987), Zhou \& Sirovich (1992), Sirovich \& Zhou (1994a,b), Webber, Handler \& Sirovich (1997), on near-wall turbulence and channel flows, in which the proper orthogonal or Karhumen-Loève decomposition (POD), first suggested for use in turbulence by Lumley (1971), is used to construct optimal bases in the

$\dagger$ Present address: Control and Dynamical Systems, California Institute of Technology, Mail Stop 107-81, 1200 E. California Blvd, Pasadena, CA, 91125, USA.

$\ddagger$ Present address: Department of Mechanical and Environmental Engineering, University of California, Santa Barbara, CA, 93106, USA. 


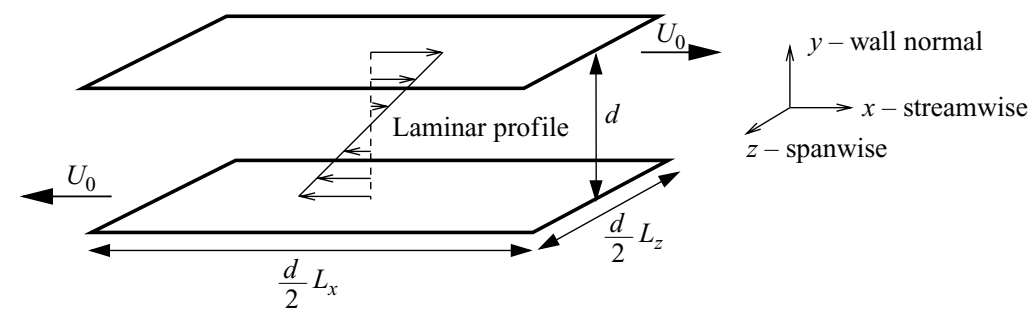

FIgURE 1. Geometry of plane Couette flow (PCF).

sense that truncations at a given order maximally capture kinetic energy on average. The Navier-Stokes equations are Galerkin projected onto low-dimensional subspaces spanned by dominant subsets of modes, yielding (relatively) tractable sets of ordinary differential equations (ODEs) for the modal amplitudes. The ODEs may then be studied to reveal interactions among coherent structures that, hopefully, form the 'core' of turbulence. Such models also suggest strategies and provide test-beds for turbulence control methods (see, e.g., Coller, Holmes \& Lumley 1994; Coller \& Holmes 1997; Smith, Moehlis \& Holmes 2003). A general account of the procedure, and relevant background information, is given in Holmes, Lumley \& Berkooz (1996). In the present paper we address perhaps the simplest turbulent flow, using it to assess the strengths and weaknesses of the low-dimensional paradigm.

We consider plane Couette flow in the minimal flow unit (MFU). Here minimality refers to the spanwise and streamwise extents of the spatial domain, which are reduced (in numerical simulations) until turbulence with reasonable statistics is just sustainable. By constraining the flow to a domain that supports only one or two coherent structures, one hopes that the dynamical interactions that sustain turbulence will be sufficiently simplified that better understanding of physical mechanisms will result. Jimenez \& Moin (1991) pioneered the idea for turbulent channel flow, and Hamilton, Kim \& Waleffe (1995) subsequently applied it to plane Couette flow. We shall draw heavily on the latter paper, hereafter referred to as HKW, in the comparative studies to follow. Parts of the present paper also parallel previous applications of POD to MFU channel flows in Webber et al. (1997) and Podvin \& Lumley (1998).

In plane Couette flow (PCF), fluid is sheared between two infinite parallel plates moving at speed $U_{0}$, in opposite directions $\pm \boldsymbol{e}_{x}$; see figure 1 . The streamwise, wallnormal, and spanwise directions are respectively $x, y$, and $z$. We non-dimensionalize lengths in units of $d / 2$ where $d$ is the gap between the plates, velocities in units of $U_{0}$, time in units of $(d / 2) / U_{0}$, and pressure in units of $U_{0}^{2} \rho$ where $\rho$ is the fluid density. Laminar flow is then given by $\boldsymbol{U}_{0}=y \boldsymbol{e}_{x},-1 \leqslant y \leqslant 1$ and the Reynolds number is $R e=U_{0} d / 2 v$, where $v$ is the kinematic viscosity. Writing $\boldsymbol{u}=\left(u_{1}, u_{2}, u_{3}\right), \boldsymbol{x}=(x, y, z)$, the evolution equation for the perturbation $(\boldsymbol{u}(\boldsymbol{x}, t), p(\boldsymbol{x}, t))$ to laminar flow becomes

$$
\frac{\partial}{\partial t} \boldsymbol{u}=-(\boldsymbol{u} \cdot \nabla) \boldsymbol{u}-y \frac{\partial}{\partial x} \boldsymbol{u}-u_{2} \boldsymbol{e}_{x}-\nabla p+\frac{1}{R e} \nabla^{2} \boldsymbol{u} .
$$

The fluid is assumed to be incompressible, i.e.

$$
\nabla \cdot \boldsymbol{u}=0,
$$

with no-slip boundary conditions at the plates, i.e.

$$
\left.\boldsymbol{u}\right|_{y= \pm 1}=0,
$$


and periodicity in the streamwise and spanwise directions, with lengths $L_{x}=1.75 \pi$ and $L_{z}=1.2 \pi$, respectively, corresponding to the MFU; see HKW. Our POD bases or empirical eigenfunctions will be derived from a direct numerical simulation (DNS) database computed at $R e=400$. In Moehlis et al. (2002) we consider the moderate aspect ratio domain $L_{x}=4 \pi, L_{z}=2 \pi$, also at $R e=400$.

Equations (1.1)-(1.3) are equivariant with respect to the following symmetries (Schmiegel 1999; Moehlis et al. 2002):

$$
\begin{aligned}
\mathscr{P} \cdot\left[\left(u_{1}, u_{2}, u_{3}, p\right)(x, y, z, t)\right] & =\left(-u_{1},-u_{2},-u_{3}, p\right)(-x,-y,-z, t), \\
\operatorname{Re} \cdot\left[\left(u_{1}, u_{2}, u_{3}, p\right)(x, y, z, t)\right] & =\left(u_{1}, u_{2},-u_{3}, p\right)(x, y,-z, t), \\
\operatorname{Re} \mathscr{P} \cdot\left[\left(u_{1}, u_{2}, u_{3}, p\right)(x, y, z, t)\right] & =\left(-u_{1},-u_{2}, u_{3}, p\right)(-x,-y, z, t), \\
\mathscr{T}_{\Delta x, \Delta z} \cdot\left[\left(u_{1}, u_{2}, u_{3}, p\right)(x, y, z, t)\right] & =\left(u_{1}, u_{2}, u_{3}, p\right)(x+\Delta x, y, z+\Delta z, t) .
\end{aligned}
$$

Thus, if $\boldsymbol{u}(\boldsymbol{x}, t)$ solves (1.1), the solution obtained by acting on $\boldsymbol{u}(\boldsymbol{x}, t)$ with any product of the actions given in equations (1.4)-(1.7) also solves it: e.g. if

$$
\left(u_{1}(x, y, z, t), u_{2}(x, y, z, t), u_{3}(x, y, z, t), p(x, y, z, t)\right)
$$

solves (1.1), then so does

$$
\left(u_{1}(x,-y, z, t),-u_{2}(x,-y, z, t), u_{3}(x,-y, z, t), p(x,-y, z, t)\right) .
$$

Physically, $\mathscr{P}$ is a point reflection about $(x, y, z)=(0,0,0)$, Re is a reflection about the plane $z=0, \operatorname{Re} \mathscr{P}$ is a rotation by $\pi$ about the $z$-axis, and $\mathscr{T}_{\Delta x, \Delta y}$ is a translation by $\Delta x$ in the streamwise direction and by $\Delta z$ in the spanwise direction.

Overall, the governing equations are equivariant with respect to the direct product $O(2) \times O(2)$, generated by the above actions (Moehlis et al. 2002). In the empiricalFourier decomposition developed below, this corresponds to independent rotations and reflections with respect to streamwise and spanwise Fourier wavenumbers. The wall layer model of Aubry et al. (1988) shares only some of these symmetries, having only $O(2) \times S^{1}$ symmetry (the upper wall is absent in Aubry et al. 1988, thus there is no analogue of $\mathscr{P}(1.4)$ ). We will use (1.4)-(1.6) in our application of the POD procedure to create a basis endowed with the appropriate symmetries, and subsequently to check that the projected ODEs preserve them.

We contend that MFU PCF turbulence is a good test case for assessment of lowdimensional modelling strategies because the turbulence is relatively mild and involves few structures (thus, if the strategy fails here, it is not likely to succeed in more fully developed turbulence), and because PCF exhibits numerous interesting properties that a model should reproduce. Specifically, the laminar state $\boldsymbol{U}_{0}$ is linearly stable for all Reynolds numbers (Drazin \& Reid 1981), but both experiments and simulations exhibit sustained turbulence for sufficiently high $\operatorname{Re}(\geqslant 380-400)$ and perturbation amplitudes (Dauchot \& Daviaud 1995a,b). Recent mathematical work (e.g. Baggett \& Trefethen 1997; Schmid \& Henningson 2000) has stressed the role of non-normal operators in such subcritical transitions (the linearized operator of 1.1 is non-normal: $L^{\dagger} L \neq L L^{\dagger}$, where ${ }^{\dagger}$ denotes adjoint), and we shall discuss this further in $\S 4.4$. Moreover, (1.1) possesses numerous branches of (unstable) steady states consisting of wavy streamwise vortices and streaks that arise in saddle-node bifurcations above $R e \approx 125$ (Nagata 1990; Clever \& Busse 1992; Schmiegel 1999), and in Schmiegel (1999) it was suggested that turbulence might be a 'chaotic repellor' formed from heteroclinic connections among such finite-amplitude solutions, as in the wall layer models of Aubry et al. (1988). The studies in Moehlis et al. (2002) support this 
conjecture. For additional information, experimental work, and references on PCF, see Bech et al. (1995) and Komminaho, Lundbladh \& Johansson (1996).

In their study, HKW identified an almost-periodic regeneration cycle or 'selfsustaining process,' in which near-wall streaks - elongated regions of spanwise alternating high- and low-speed fluid - are produced by streamwise vortices in a process previously discussed in Kline (1967). The streaks, almost straight initially, develop streamwise waviness as a result of a linear instability; they then break down and, in doing so, regenerate the streamwise vortices, after which the process begins anew. It has been suggested that such a self-sustaining process is a universal characteristic of shear flow turbulence (Hamilton et al. 1995; Waleffe 1997), cf. Reddy et al. (1998) and Jiménez \& Pinelli (1999).

We develop two classes of models in this paper, using expansions in terms of 'coupled' and 'uncoupled' modes. The former employs the vector-valued basis functions (empirical eigenfunctions) delivered directly by the POD, as described in Holmes et al. (1996). However, as pointed out by Moffatt (1990), and acknowledged in Holmes (1990) and Moehlis et al. (2002), this can lead to paradoxical results in which, for example, flows containing only streamwise-invariant modes can extract energy from uniform mean shears such as the laminar Couette solution $\boldsymbol{U}_{0}$. In Berkooz et al. (1991) it was suggested that decoupling of streamwise and spanwise velocity components might repair this situation, and here we use a generalization of this notion due to Waleffe (1995b) to develop models with suitably uncoupled modes. In both cases we use Heisenberg (eddy viscosity) type models to account for energy transfer to the (many) neglected modes. We concentrate on truly low-dimensional models which include modes with streamwise variation. Noteworthy POD-Galerkin models for the turbulent boundary layer which include modes with streamwise variation were obtained by Sanghi \& Aubry (1993) and Sirovich \& Zhou (1994a), the latter constructed to be very low-dimensional and the former testing the effect of the inclusion of modes with streamwise variation on the streamwise-invariant low-dimensional model of Aubry et al. (1988).

In $\S 2$ we briefly describe the DNS data and the POD procedure and outline properties of the empirical eigenvalues derived from it. Sections 3-4 form the heart of the paper; here models based on coupled and uncoupled modes are derived and comparisons with the DNS data made and discussed. In $\S 5$ we conclude, and compare our models with other low-dimensional models, including those of Waleffe (1995a, $b$, 1997) (following HKW) and Eckhardt \& Mersmann (1999) for shear flow turbulence. The thesis of Smith (2003) contains many additional details and analyses.

\section{The database, the POD, and empirical eigenfunctions}

\subsection{The numerical method and database}

Following HKW and Kawahara \& Kida (2001), we used a modification of the usual velocity-vorticity algorithm for channel flow (Kim, Moin \& Moser 1987). As in HKW, convective terms were advanced by means of a third-order Runge-Kutta routine (Zang \& Hussaini 1985; Peyret 2002), rather than the original second-order AdamsBashforth method. De-aliased Fourier expansions were employed in the streamwise and spanwise directions and Chebyshev polynomials in the wall-normal direction. A computational grid of $16 \times 33 \times 16$ (streamwise $\times$ wall-normal $\times$ spanwise) was used. To produce the simulation data, we began with random initial conditions at $R e=625$, allowed the system to converge on the turbulent state, and used the final states as new initial conditions as $R e$ was successively reduced to 400 in decreasing steps. Our 

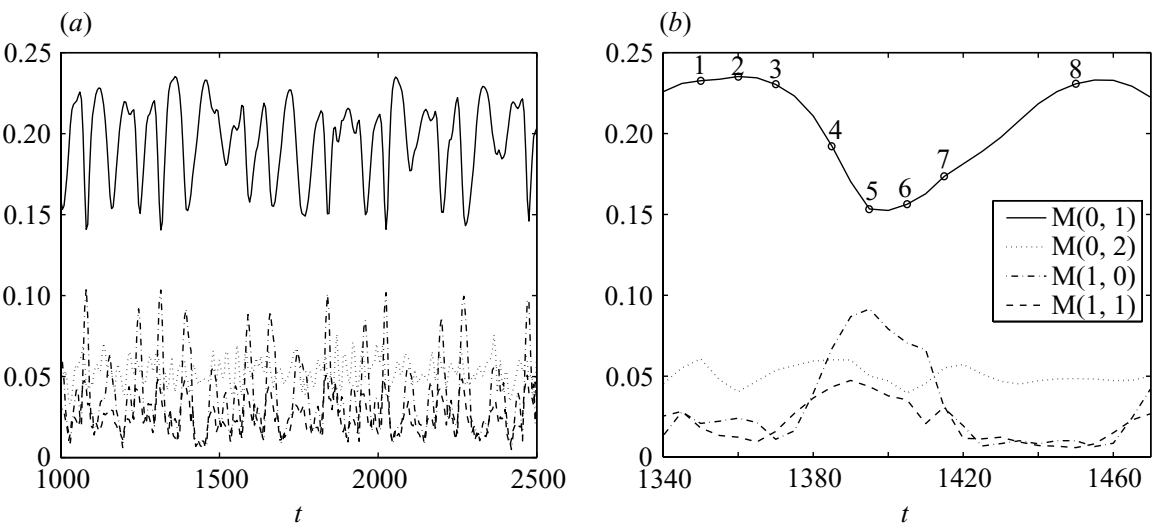

FIGURE 2. The behaviour of the RMS modal velocities, as defined by (2.1), for several wavenumber pairs: $(a)$ over 1500 time units of the DNS, $(b)$ a close-up of one representative cycle. Here, and for similar plots throughout this paper, the legend in $(b)$ also applies to $(a)$.

code was modified from a channel flow code kindly provided by C. W. Rowley, and validated for PCF by comparison with statistics of HKW and Kawahara \& Kida (2001), obtaining agreements within $2.2 \%$ for mean velocity and $1.2 \%$ for RMS fluctuations (in $L^{2}$ norm).

After allowing transients to decay and a statistically stationary (turbulent) state to become established, we ran for 20000 non-dimensional time units, assembling a database of 4000 velocity field snapshots $\left\{\boldsymbol{u}\left(\boldsymbol{x}, t_{j}\right)\right\}$ by recording every 500th timestep $(\Delta t=0.01)$. To ensure that the ensemble possesses the appropriate discrete symmetries (1.4)-(1.6), we then quadrupled the number of samples in the database by applying these symmetry operations to create our ensemble. This ensures that the POD modes share the symmetries of the governing equations, even if the 'raw' database does not. See Berkooz \& Titi (1993), Aubry, Lian \& Titi (1993), Moehlis et al. (2002) and Smith, Moehlis \& Holmes (2004).

\subsection{Phenomenology of turbulence in the PCF-MFU}

With a view to comparing velocity fields reconstructed from low-dimensional models with 'full' DNS data, we briefly survey the results of HKW and present analogous results from our DNS. In HKW the RMS modal velocities are defined as

$$
\mathrm{M}\left(n_{x}, n_{z}\right) \stackrel{\text { def }}{=}\left(\int_{-1}^{1}\left[\tilde{u}_{1}^{2}\left(n_{x}, y, n_{z}\right)+\tilde{u}_{2}^{2}\left(n_{x}, y, n_{z}\right)+\tilde{u}_{3}^{2}\left(n_{x}, y, n_{z}\right)\right] \mathrm{d} y\right)^{1 / 2},
$$

where the tildes represent Fourier mode amplitudes, and the temporal behaviour of this quantity for various wavenumber pairs $\left(n_{x}, n_{z}\right)$ is studied. Approximately periodic dynamics are found for certain (dominant) modal velocities; in particular, $M(0,1)$ and $\mathrm{M}(1,0)$ remain in near antiphase: peaks (troughs) in the former often being accompanied by troughs (peaks) in the latter: figure 2 (cf. figure 3(a) of HKW). This figure also shows that the temporal dynamics of $\mathrm{M}(1,1)$ is much the same as that of $\mathrm{M}(1,0)$, with a recurrence period of 80-100 non-dimensional time units, while $\mathrm{M}(0,2)$ is less regular. In Sirovich \& Zhou (1994b), Webber et al. (1997) and references therein, the $n_{x}=0$ and $n_{x} \neq 0$ modes are respectively called 'roll' and 'propagating' and it is shown (Webber et al. 1997, figure 15) that in energetic events 

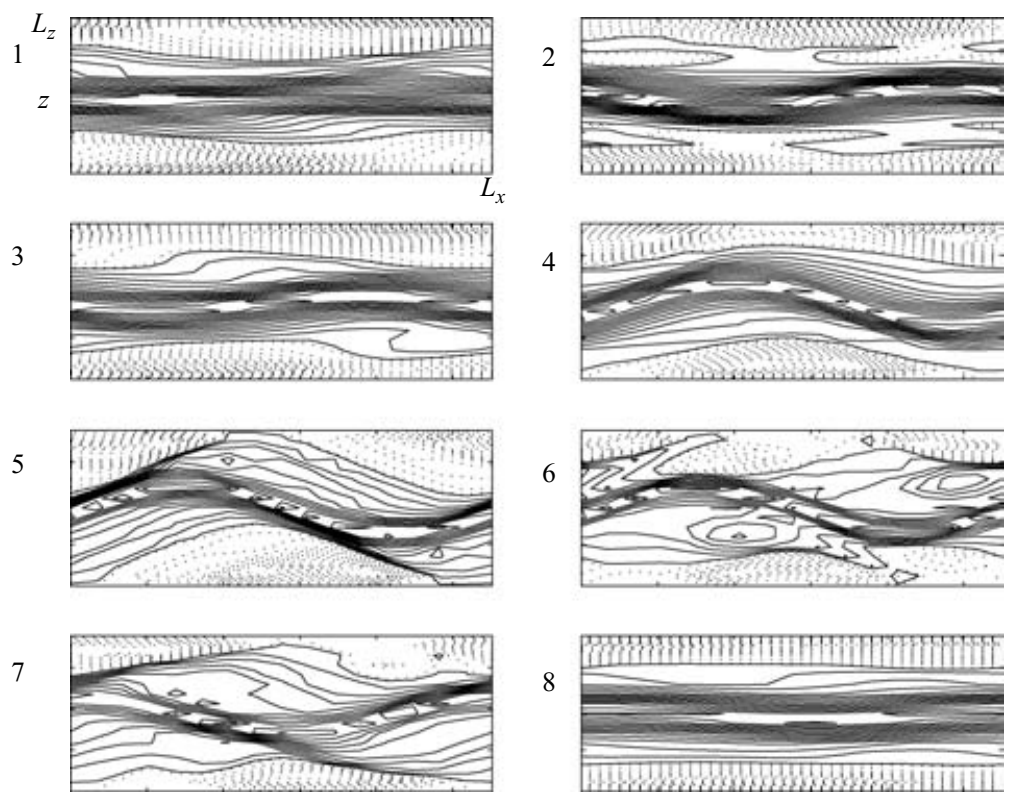

FIGURE 3. The streak breakdown process in DNS simulations of PCF in the MFU as indicated by contours of $u$ (solid positive, dashed negative) in the $(x, z)$-plane lying between the two plates. Here, and for similar plots in this paper, we label the axes in the subplot in the upper left-hand corner only. Since the laminar solution is identically zero in this mid-plane it makes no contribution to the contours in this plot.

(a)

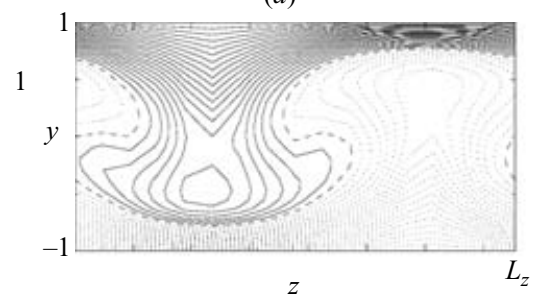

5

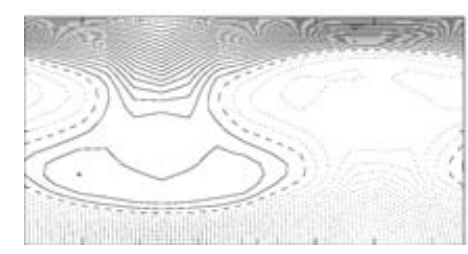

(b)
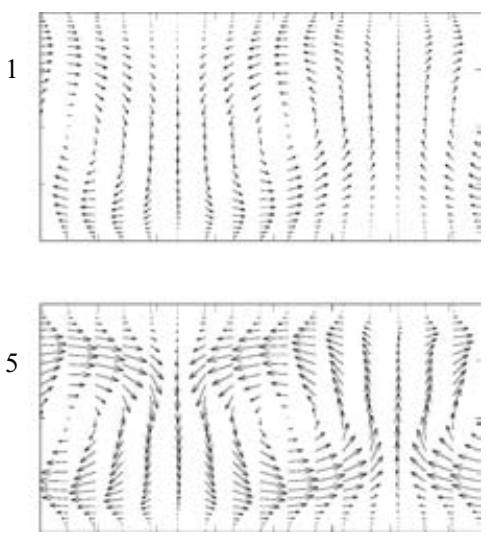

FIGURE 4. (a) Streamwise velocity contours and $(b)$ cross-stream velocity vectors associated with streamwise-invariant modes before breakdown (1) and after breakdown (5).

the roll modes intensify and then collapse, followed, in near-antiphase, by the rise and fall of the propagating modes, much as in figure 2.

Figure 3 (cf. figure 2 of $\mathrm{HKW}$ ) shows mid-plane contours of the streamwise velocity at the times $1-8$ marked on the $M(0,1)$ curve in figure 2 . At 1 , the flow shows prominent streaks. The flow pattern then develops greater variation with respect to streamwise position, until at 5 the streaks break down. They then regenerate and at 8 the process begins anew. We show average streamwise velocity contours and cross-stream velocity vectors in the cross-stream plane in figure 4. To simplify the 
representation of these quantities we consider contributions from the streamwiseinvariant modes only (cf. figure 4 of HKW). Here 'before' and 'after' breakdown correspond to the points 1 and 5 in figure 2. This plot shows that the contours of streamwise velocity are much the same after breakdown as before, though perhaps a little more diffuse. We also note that the cross-stream velocities increase during the breakdown process, as explained in HKW.

The streamwise velocity contours presented in figure 4 include the laminar solution $\boldsymbol{U}_{0}$. Versions of this plot in which the laminar solution is excluded are subsequently given in comparing these results with those of low-dimensional models.

\subsection{The proper orthogonal decomposition}

Numerous accounts of the POD are available, notably those of Sirovich (1987), Berkooz, Holmes \& Lumley (1993) and Holmes et al. (1996); rather than describing the procedure in detail again, we refer the reader to those references, our earlier paper on PCF (Moehlis et al. 2002), and the tutorial Smith et al. (2004). Here we merely recall that the POD modes are the eigenfunctions of the integral operator equation

$$
\sum_{j=1}^{3} \iiint_{\Omega}\left\langle u_{i}(\boldsymbol{x}, t) u_{j}^{*}\left(\boldsymbol{x}^{\prime}, t\right)\right\rangle \Phi_{j}^{(n)}\left(\boldsymbol{x}^{\prime}\right) \mathrm{d}^{3} \boldsymbol{x}^{\prime}=\lambda_{n_{x}, n_{z}}^{(n)} \Phi_{i}^{(n)}(\boldsymbol{x}),
$$

where $\Omega$ denotes the flow domain, $\left\langle u_{i}(\boldsymbol{x}, t) u_{j}^{*}\left(\boldsymbol{x}^{\prime}, t\right)\right\rangle$ the ensemble averaged autocorrelation tensor, and $\boldsymbol{\Phi}^{(n)}=\left(\Phi_{1}^{(n)}, \Phi_{2}^{(n)}, \Phi_{3}^{(n)}\right)$ the POD modes, indexed by 'quantum numbers' $n \in Z^{+}$and streamwise and spanwise wavenumbers $n_{x}, n_{z}$. Translation invariance (1.7) implies that Fourier decompositions in the latter directions are optimal (Holmes et al. 1996), and hence that we may write

$$
\boldsymbol{\Phi}^{(n)}(\boldsymbol{x})=\sum_{n_{x}, n_{z}} \boldsymbol{\Phi}_{n_{x}, n_{z}}^{(n)}(\boldsymbol{x})=\frac{1}{\sqrt{L_{x} L_{z}}} \sum_{n_{x}, n_{z}} \exp \left(2 \pi \mathrm{i}\left(\frac{n_{x} x}{L_{x}}+\frac{n_{z} z}{L_{z}}\right)\right) \boldsymbol{\phi}_{n_{x}, n_{z}}^{(n)}(y) .
$$

The representation of the velocity field is therefore

$$
\boldsymbol{u}(\boldsymbol{x}, t)=\frac{1}{\sqrt{L_{x} L_{z}}} \sum_{n} \sum_{n_{x}} \sum_{n_{z}} a_{n_{x}, n_{z}}^{(n)}(t) \exp \left(2 \pi \mathrm{i}\left(\frac{n_{x} x}{L_{x}}+\frac{n_{z} z}{L_{z}}\right)\right) \boldsymbol{\phi}_{n_{x}, n_{z}}^{(n)}(y) .
$$

The modal coefficients $a_{n_{x}, n_{z}}^{(n)}$ are complex unless $n_{x}=n_{z}=0$. However, reality of $\boldsymbol{u}$ and $\boldsymbol{\Phi}^{(n)}(\boldsymbol{x})$ implies

$$
a_{n_{x}, n_{z}}^{(n)}(t)=a_{-n_{x},-n_{z}}^{(n)^{*}}(t) \quad \text { and } \quad \boldsymbol{\phi}_{n_{x}, n_{z}}^{(n)}(y)=\boldsymbol{\phi}_{-n_{x},-n_{z}}^{(n)^{*}}(y) .
$$

As noted in the introduction, we shall refer to $\boldsymbol{\phi}_{n_{x}, n_{z}}^{(n)}(y)$ as the $\left(n, n_{x}, n_{z}\right)$ coupled POD mode, to underline the fact that the relative magnitudes of all three velocity components are fixed by the vector $\boldsymbol{\phi}_{n_{x}, n_{z}}^{(n)}(y)$.

A lengthy analysis, detailed in Smith (2003) and Smith et al. (2004), then allows us to deduce the following actions of $\mathscr{P}$ and $\mathrm{Re}$ on the modal amplitude coefficients $a_{n_{x}, n_{2}}^{(n)}$ :

$$
\begin{aligned}
\mathscr{P} \cdot a_{n_{x}, n_{z}}^{(n)}(t) & =c_{\mathscr{P}} a_{-n_{x},-n_{z}}^{(n)}(t), \\
\operatorname{Re} \cdot a_{n_{x}, n_{z}}^{(n)}(t) & =c_{\operatorname{Re}} a_{n_{x},-n_{z}}^{(n)}(t), \\
\operatorname{Re} \mathscr{P} \cdot a_{n_{x}, n_{z}}^{(n)}(t) & =c_{\mathscr{P}} c_{\operatorname{Re}} a_{-n_{x}, n_{z}}^{(n)}(t),
\end{aligned}
$$




$\begin{array}{ccc}\left(n, n_{x}, n_{z}\right) & \lambda_{n_{x}, n_{z}}^{(n)} & \% E_{n_{x}, n_{z}}^{(n)} \\ (1,0,0) & 4.4550 & 68.02 \\ (1,0, \pm 1) & 0.7821 & 23.88 \\ (1,0, \pm 2) & 0.0543 & 1.66 \\ (1, \pm 1,0) & 0.0386 & 1.18 \\ (1,0, \pm 3) & 0.0195 & 0.59 \\ (2,0,0) & 0.0174 & 0.27 \\ (2,0, \pm 1) & 0.0123 & 0.38 \\ (1, \pm 1, \pm 2) & 0.0109 & 0.33 \\ (1, \pm 1, \pm 1) & 0.0090 & 0.27 \\ (3,0,0) & 0.0068 & 0.10 \\ (4,0,0) & 0.0054 & 0.08 \\ (3,0, \pm 1) & 0.0039 & 0.12\end{array}$

TABLE 1. Eigenvalues for the POD modes for PCF in the MFU.

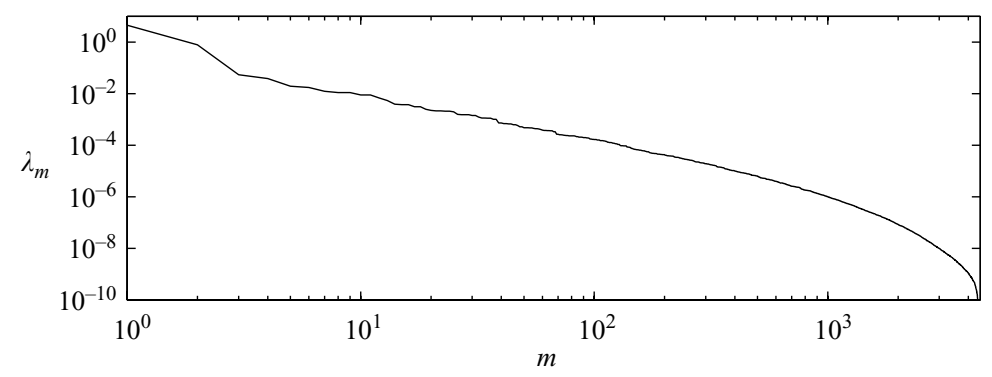

Figure 5. Spectrum of the largest 4500 eigenvalues found through POD analysis of PCF in the MFU ordered by magnitude.

where

$$
\begin{aligned}
& c_{\mathscr{P}}= \begin{cases}-1 & \text { if } n_{x}=n_{z}=0 \text { and } \phi_{0,0}^{(n)} \text { has components even in } y \\
+1 & \text { otherwise, }\end{cases} \\
& c_{\operatorname{Re}}= \begin{cases}-1 & \text { if } n_{x}=n_{z}=0 \text { and } \phi_{3,0,0}^{(n)}=0 \\
-1 & \text { if } n_{z}=0 \text { and } \phi_{1, n_{x}, 0}^{(n)}=\phi_{2, n_{x}, 0}^{(n)}=0 \\
+1 & \text { otherwise. }\end{cases}
\end{aligned}
$$

The eigenvalues $\lambda_{n_{x}, n_{z}}^{(n)}$ are equal to twice the average kinetic energy carried by the corresponding modes, i.e. $\lambda_{n_{x}, n_{z}}^{(n)}=\left\langle\left|\hat{a}_{n_{x}, n_{z}}^{(n)}\right|^{2}\right\rangle$ where $\hat{a}_{n_{x}, n_{z}}^{(n)}$ is the 'true' modal amplitude obtained by projection of the DNS data onto the $\left(n, n_{x}, n_{z}\right)$ mode. The decay properties of these eigenvalues therefore indicate which modes should be included on energetic grounds. Table 1 shows the eigenvalues associated with the first twelve (sets of) POD modes in order of decreasing eigenvalue magnitude. Here

$$
\% E_{n_{x}, n_{z}}^{(n)} \stackrel{\text { def }}{=}\left(\lambda_{n_{x}, n_{z}}^{(n)} / \sum_{m, m_{x}, m_{z}} \lambda_{m_{x}, m_{z}}^{(m)}\right) \times 100
$$

is the percentage of average total energy contained in the $\left(n, n_{x}, n_{z}\right)$ POD mode. The symmetries guarantee that $\lambda_{n_{x}, n_{z}}^{(n)}=\lambda_{n_{x},-n_{z}}^{(n)}$, and we lump these modes together accordingly. (Hence the $(2,0, \pm 1)$ POD modes, each with eigenvalue of 0.0123 , together account for more kinetic energy on average than the $(2,0,0)$ mode with eigenvalue 0.0174.) Figure 5 summarizes the eigenvalue decay properties for the 

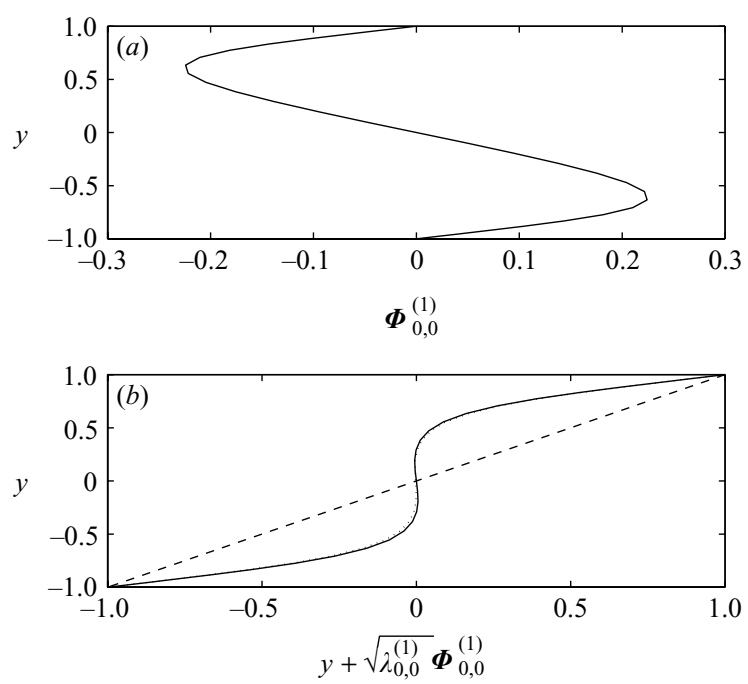

Figure 6. (a) The $x$-component of the POD mode $\boldsymbol{\Phi}_{0,0}^{(1)}$. The $y$ - and $z$-components are equal to zero. (b) The velocity profile obtained by adding this POD mode with RMS amplitude $\sqrt{\lambda_{0,0}^{(1)}}=\sqrt{\left\langle\left|\hat{a}_{0,0}^{(1)}(t)\right|^{2}\right\rangle}$ to the laminar state $\boldsymbol{U}_{0}=z \boldsymbol{e}_{x}$. The mean flow obtained from the full DNS ensemble average is also indicated (dotted curve, barely discernible).

first 4500 modes. While it suggests inertial range scaling similar to that observed by Knight \& Sirovich (1990), we do not believe that substantial conclusions can be drawn from these low Reynolds number data.

The three most energetic modes have Fourier wavenumbers $\left(n_{x}, n_{z}\right)=(0,0),(0,1)$, $(0,2)$; a similar triad appeared in the moderate aspect ratio PCF study of Moehlis et al. (2002). Interestingly, the fourth most energetic mode is the spanwise-invariant $(1,1,0)$ mode, which has neither a streamwise nor a wall-normal component and is thus unable to interact directly with the $(1,0,0)$ mode representing the mean flow. In figure 6 we show the $(1,0,0)$ mode and indicate its close approximation to the full (DNS) mean velocity profile. The four 'two-dimensional modes' that follow the $(1,0,0)$ mode in table 1 are plotted in figure 7 . Over $90 \%$ of the turbulent kinetic energy is captured by the first two modes, while $99 \%$ is captured by the leading 43 modes. Table 1 agrees well with independent results of Gibson (2002).

The large percentage of energy contained in the $(1,0,0)$ mode illustrates the important effect that turbulent fluctuations have on the mean profile, cf. Moehlis, Faisst \& Eckhardt (2004). We also note that it is not surprising that streamwise vortices and their associated streaks, i.e. $\left(n, 0, \pm n_{z}\right)$ modes with $n_{x}=0$, are dominant coherent structures for turbulent PCF. Similar structures have been found numerically as unstable steady solutions of the full evolution equations (Nagata 1990; Clever \& Busse 1992; Schmiegel 1999); these finite-amplitude solutions have some variation in the streamwise direction, but nonetheless strongly resemble $n_{x}=0$ POD modes, which can be seen as streamwise-averaged versions of them. Furthermore, streamwise vortices and associated streaks can be stabilized by spatially forcing PCF with a stationary wire or bead (Dauchot \& Daviaud 1995b; Bottin, Dauchot \& Daviaud 1997; Bottin et al. 1998; Barkley \& Tuckerman 1999), they are involved in the selfsustaining process elucidated in HKW and Waleffe (1997), they can exhibit substantial transient growth due to linear effects (Butler \& Farrell 1992), and they are ubiquitous 

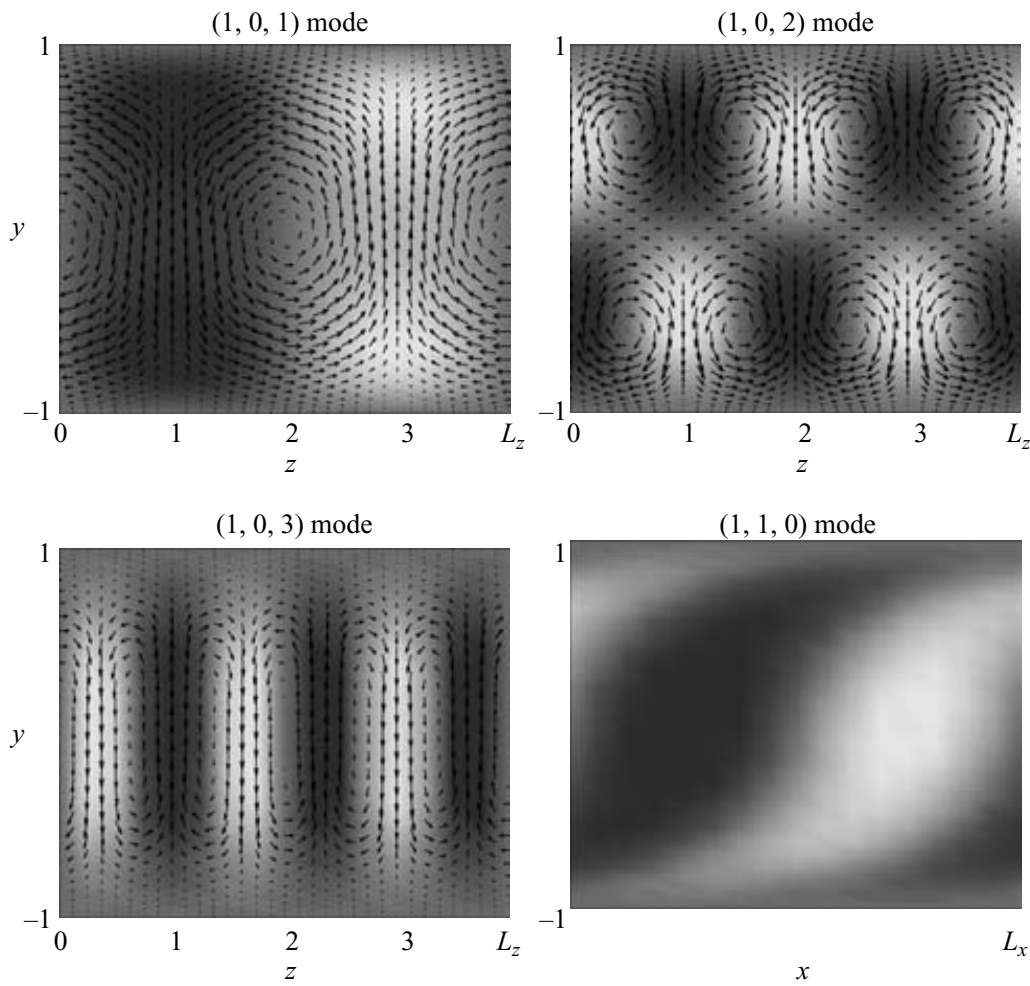

FIGURE 7. Flow fields $\boldsymbol{u}$ associated with the $\left(n, n_{x}, n_{z}\right)=(1,0,1),(1,0,2),(1,0,3)$ and $(1,1,0)$ POD modes. For the $(1,0,1),(1,0,2)$ and $(1,0,3)$ POD modes the vectors show the spanwise and wall-normal velocities, while the dark (light) shading denotes positive (negative) streamwise velocity. For the $(1,1,0)$ mode the $u$ and $v$ components are identically zero, and the dark (light) shading now denotes positive (negative) spanwise velocity.

in wall-bounded flows (Holmes et al. 1996). The $(1,1,1)$ and $(1,1,2)$ modes consist of vortices whose axes are parallel to the walls but do not point in the streamwise direction, and are analogous to spirals for Taylor-Couette flow.

\section{Low-dimensional models for the MFU using coupled modes}

We first briefly describe the general derivation and some properties of the ODEs resulting from Galerkin projection of the Navier-Stokes equations onto subspaces spanned by sets of the POD modes obtained above. Inserting (2.4) into (1.1) and performing a Galerkin projection, we obtain ODEs of the form

$$
\begin{gathered}
\dot{a}_{n_{x}, n_{z}}^{(n)}=\sum_{k=1}^{\infty} \hat{A}_{n_{x}, n_{z}}^{(n, k)} a_{n_{x}, n_{z}}^{(k)}+[N(\boldsymbol{a}, \boldsymbol{a})]_{n, n_{x}, n_{z}}, \\
n=1,2, \cdots, \quad n_{x}, n_{z}=\cdots,-2,-1,0,1,2, \cdots,
\end{gathered}
$$

where

$$
[N(\boldsymbol{a}, \boldsymbol{a})]_{n, n_{x}, n_{z}} \stackrel{\text { def }}{=} \sum_{\substack{m, k \\ m_{x}, m_{z}}} \hat{\boldsymbol{B}}_{n_{x}, n_{z} m_{x} m_{z}}^{(n, m, k)} a_{m_{x}, m_{z}}^{(m)} a_{n_{x}-m_{x}, n_{z}-m_{z}}^{(k)},
$$


and, letting a prime denote differentiation with respect to $z$,

$$
\begin{aligned}
\hat{A}_{n_{x}, n_{z}}^{(n, k) \stackrel{\text { def }}{=}} & -\frac{1}{R e}\left(\left(\frac{2 \pi n_{x}}{L_{x}}\right)^{2}+\left(\frac{2 \pi n_{z}}{L_{z}}\right)^{2}\right) \delta_{n k} \\
& -\int_{-1}^{1} \phi_{2, n_{x}, n_{z}}^{(n)} \phi_{1, n_{x}, n_{z}}^{(k) *} \mathrm{~d} y-\frac{2 \pi \mathrm{i} n_{x}}{L_{x}} \sum_{j=1}^{3} \int_{-1}^{1} y \phi_{j, n_{x}, n_{z}}^{(n)} \phi_{j, n_{x}, n_{z}}^{(k) *} \mathrm{~d} y \\
& -\frac{1}{R e} \sum_{j=1}^{3} \int_{-1}^{1} \phi_{j, n_{x}, n_{z}}^{(n)^{\prime}} \phi_{j, n_{x}, n_{z}}^{(k) *^{\prime}} \mathrm{d} y, \\
\hat{B}_{n_{x}, n_{z} m_{x} m_{z}}^{(n, m, k)} \stackrel{-}{\frac{1}{=}} & \frac{1}{\sqrt{L_{x} L_{z}}} \sum_{j=1}^{3} \int_{-1}^{1}\left(\frac{2 \pi \mathrm{i} m_{x}}{L_{x}} \phi_{1, n_{x}-m_{x}, n_{z}-m_{z}}^{(k)} \phi_{j m_{x} m_{z}}^{(m)}\right. \\
+ & \left.\phi_{2, n_{x}-m_{x}, n_{z}-m_{z}}^{(k)} \phi_{j m_{x} m_{z}}^{(m)^{\prime}}+\frac{2 \pi \mathrm{i} m_{z}}{L_{z}} \phi_{3, n_{x}-m_{x}, n_{z}-m_{z}}^{(k)} \phi_{j m_{x} m_{z}}^{(m)}\right) \phi_{j, n_{x}, n_{z}}^{(n) *} \mathrm{~d} y .
\end{aligned}
$$

The projected ODEs (3.1) are equivariant with respect to the group actions (2.6)(2.8) and the continuous symmetries

$$
\begin{aligned}
\mathscr{T}_{\Delta x}: a_{n_{x}, n_{z}}^{(n)}(t) \mapsto \mathrm{e}^{\mathrm{i} n_{x} \phi_{x}} a_{n_{x}, n_{z}}^{(n)}(t), \\
\mathscr{T}_{\Delta z}: a_{n_{x}, n_{z}}^{(n)}(t) \mapsto \mathrm{e}^{\mathrm{i} n_{z} \phi_{z}} a_{n_{x}, n_{z}}^{(n)}(t),
\end{aligned}
$$

where $\phi_{x}=2 \pi \Delta x / L_{x}$ and $\phi_{z}=2 \pi \Delta z / L_{z}$; i.e. writing (3.1) as $\dot{\boldsymbol{a}}=f(\boldsymbol{a})$, it is necessary that $f(\gamma \boldsymbol{a})=\gamma f(\boldsymbol{a})$ for all $\gamma \in O(2) \times O(2)$. This implies that many terms which might appear in (3.1), in fact, vanish. Also, by exploiting symmetry properties of the POD modes (specifically, oddness or evenness of components $\phi_{i, n_{x}, n_{z}}^{(n)}$ under $y \rightarrow-y$ ), it can be shown that certain of the $\hat{A}$ and $\hat{B}$ vanish identically. Finally, the nonlinear terms in the Navier-Stokes equations are energy-conserving. As shown, e.g., in Moehlis et al. (2002), this implies that

$$
\sum_{n=1}^{\infty} \sum_{n_{x}=-\infty}^{\infty} \sum_{n_{z}=-\infty}^{\infty} a_{n_{x}, n_{z}}^{(n)^{*}}[N(\boldsymbol{a}, \boldsymbol{a})]_{n, n_{x}, n_{z}}=0 .
$$

Equation (3.6) and the symmetries (2.6)-(2.8) and (3.4)-(3.5) provide checks on the numerically computed nonlinear coefficients: in all cases we have found that (3.6) is satisfied to $0.01 \%$, and we have therefore subsequently rounded off the coefficients to ensure that these symmetries are exactly respected.

The ODEs (3.1) are similar to those of the turbulent boundary layer problem in Aubry et al. (1988), with the following important differences. In Aubry et al. (1988), in place of $\boldsymbol{U}_{0}=y \boldsymbol{e}_{x}$, the analogue of (1.1) involves a spatially averaged ( $t$ dependent) mean turbulent velocity, modelled as a balance between the effects of pressure and those of the coherent structures, giving cubic terms in the ODEs. No such modelling is required here: the nonlinear terms derive directly from 1.1 and the $n_{x}=n_{z}=0$ modes represent time-varying turbulent modifications to the mean. Second, the contribution from the pressure term at the outer edge of the wall layer was modelled as stochastic forcing in Aubry et al. (1988); here, it makes no contribution because of the divergence-free expansion (2.4) and no-slip and periodic conditions at the boundaries of $\Omega$ (Holmes et al. 1996). Finally, in Aubry et al. (1988) the ODEs are equivariant under $O(2) \times S^{1}$; here, the additional reflection and rotation symmetries make the ODEs equivariant under $O(2) \times O(2)$, further constraining the modal interactions. 

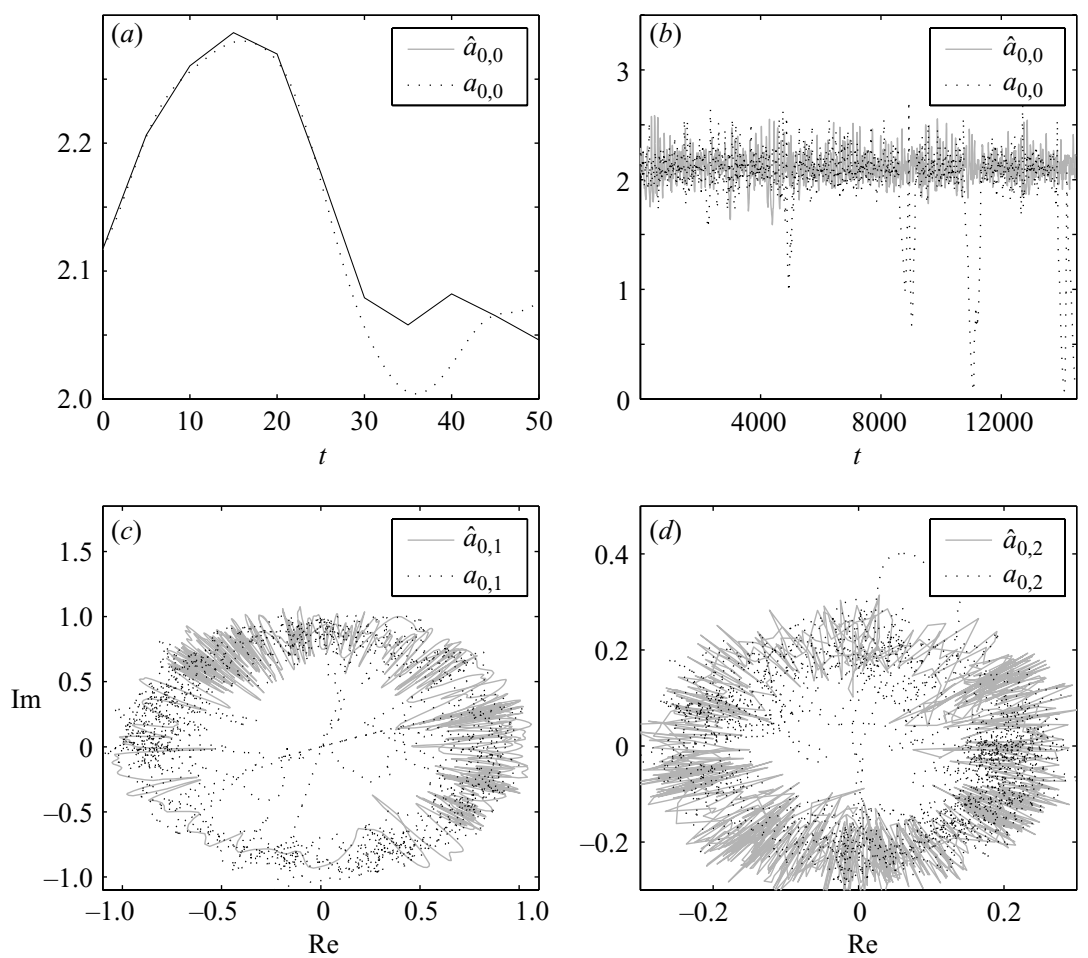

FIGURE 8. Short-term tracking and phase-space geometry for a high-dimensional POD-based model of plane Couette flow (PCF). In this and similar figures to follow a comparison is made between the modal amplitude, $a_{n_{x}, n_{z}}^{(n)}$, obtained from integration of the low-dimensional model and the 'true' modal amplitude, $\hat{a}_{n_{x}, n_{z}}^{(n)}$, obtained from projection of the DNS data onto the $\left(n, n_{x}, n_{z}\right)$ mode. We drop the superscript (1) from all modal amplitudes in the legend for clarify. $(a, b)$ Short and long time histories of $(1,0,0)$ 'mean flow' modes for DNS (solid) and model (dotted). $(c, d)$ Projections of DNS (solid) and model (dotted) solutions onto the complex $(1,0, \pm 1)$ and $(1,0, \pm 2)$ modal planes.

\subsection{High-dimensional truncations}

While we are primarily interested in deriving (very) low-dimensional models by the POD-Galerkin procedure, it is of interest first to determine how relatively large truncations capture the detailed dynamics of the DNS database, as in Moehlis et al. (2002). To this end, in figure 8 we present a numerical simulation of a model containing the 600 most energetic POD modes. This achieves good short-term tracking for $\approx 20$ non-dimensional time units, after which sensitive dependence on initial conditions inevitably drives the model and DNS trajectories apart, although the long-term statistics of the 12 most energetic modes are reproduced well. In figure $8(c, d)$ (cf. figures 9 and 14, below) we show projections of the model and DNS solutions onto the two most energetic modes $(1,0, \pm 1),(1,0, \pm 2)$ along with the mean $(1,0,0)$, illustrating that the model modes display similar behaviour to the DNS modes, with occasional (unphysical) excursions towards the laminar state, corresponding to near-relaminarization of the flow. We note that this effect disappears once $\approx 1000$ modes are retained.

John Gibson, who independently studied the same problem in Gibson (2002), also determined that approximately 1000 modes were required to faithfully reproduce the DNS behaviour. 


\subsection{Structure of the projected ODEs}

\subsubsection{The 9-coupled-mode model: dynamical equations}

We first display the equations resulting from a projection which includes all $n=1$ modes in table $1((1,0,0),(1,0,1),(1,0,2),(1,1,0),(1,1, \pm 1),(1,0,3)$, and $(1,1, \pm 2))$. This will encompass all the models considered in this section. Here the ODEs (3.1) take the particular form:

$$
\begin{aligned}
& \dot{a}_{0,0}^{(1)}=A_{0,0} a_{0,0}^{(1)}+2\left(\left.\left|B_{0,1}\right| a_{0,1}^{(1)}\right|^{2}+B_{0,2}\left|a_{0,2}^{(1)}\right|^{2}+B_{0,3}\left|a_{0,3}^{(1)}\right|^{2}\right. \\
& \left.+B_{1,1}\left(\left|a_{1,1}^{(1)}\right|^{2}+\left|a_{1,-1}^{(1)}\right|^{2}\right)+B_{1,2}\left(\left|a_{1,2}^{(1)}\right|^{2}+\left|a_{1,-2}^{(1)}\right|^{2}\right)\right) \text {, } \\
& \dot{a}_{0,1}^{(1)}=A_{0,1} a_{0,1}^{(1)}-B_{0,1} a_{0,0}^{(1)} a_{0,1}^{(1)} \\
& -{ }^{0,1} C_{0,1}^{0,2} a_{0,2}^{(1)} a_{0,1}^{(1) *}+\left({ }^{0,1} C_{1,-1}^{1,0}-{ }^{0,1} C_{1,0}^{1,1}\right) a_{1,1}^{(1)} a_{1,0}^{(1) *} \\
& -\left({ }^{0,1} C_{1,-2}^{1,-1}+{ }^{0,1} C_{1,1}^{1,2}\right) a_{1,-1}^{(1)} a_{1,-2}^{(1) *}-\left({ }^{0,1} C_{0,2}^{0,3}+{ }^{0,1} C_{0,-3}^{0,-2}\right) a_{0,3}^{(1)} a_{0,2}^{(1) *} \\
& +\left({ }^{0,1} C_{1,1}^{1,2}+{ }^{0,1} C_{1,-2}^{1,-1}\right) a_{1,2}^{(1)} a_{1,1}^{(1) *}+\left({ }^{0,1} C_{1,0}^{1,1}-{ }^{0,1} C_{1,-1}^{1,0}\right) a_{1,0}^{(1)} a_{1,-1}^{(1) *}, \\
& \dot{a}_{0,2}^{(1)}=A_{0,2} a_{0,2}^{(1)}-B_{0,2} a_{0,0}^{(1)} a_{0,2}^{(1)} \\
& -\left({ }^{0,2} C_{1,-2}^{1,0}+{ }^{0,2} C_{1,0}^{1,2}\right) a_{1,0}^{(1)} a_{1,-2}^{(1) *}+{ }^{0,1} C_{0,1}^{0,2}\left(a_{0,1}^{(1)}\right)^{2}-2{ }^{0,2} C_{1,-1}^{1,1} a_{1,1}^{(1)} a_{1,-1}^{(1)} \\
& +\left({ }^{0,1} C_{0,-3}^{0,-2}-{ }^{0,2} C_{0,1}^{0,3}\right) a_{0,3}^{(1)} a_{0,1}^{(1) *}-\left({ }^{0,2} C_{1,0}^{1,2}+{ }^{0,2} C_{1,-2}^{1,0}\right) a_{1,2}^{(1)} a_{1,0}^{(1) *}, \\
& \dot{a}_{1,0}^{(1)}=A_{1,0} a_{1,0}^{(1)} \\
& +\left({ }^{0,1} C_{1,-1}^{1,0}-{ }^{1,0} C_{0,1}^{1,-1}\right) a_{0,1}^{(1)} a_{1,-1}^{(1)}+\left({ }^{0,2} C_{1,-2}^{1,0}+{ }^{1,0} C_{0,2}^{1,-2}\right) a_{0,2}^{(1)} a_{1,-2}^{(1)} \\
& +\left({ }^{1,0} C_{0,1}^{1,-1}-{ }^{0,1} C_{1,-1}^{1,0}\right) a_{1,1}^{(1)} a_{0,1}^{(1) *}+\left({ }^{1,0} C_{0,2}^{1,-2}+{ }^{0,2} C_{1,-2}^{1,0}\right) a_{1,2}^{(1)} a_{0,2}^{(1) *}, \\
& \dot{a}_{1,1}^{(1)}=A_{1,1} a_{1,1}^{(1)}-B_{1,1} a_{0,0}^{(1)} a_{1,1}^{(1)} \\
& +\left({ }^{0,1} C_{1,0}^{1,1}-{ }^{1,0} C_{0,1}^{1,-1}\right) a_{0,1}^{(1)} a_{1,0}^{(1)}+{ }^{0,1} C_{0,2}^{0,3} a_{0,2}^{(1)} a_{1,-1}^{(1)} \\
& +\left({ }^{1,1} C_{0,-3}^{1,-2}+{ }^{1,1} C_{-1,2}^{0,3}\right) a_{0,3}^{(1)} a_{1,-2}^{(1)}-\left({ }^{1,1} C_{0,1}^{1,2}+{ }^{0,1} C_{1,-2}^{1,-1}\right) a_{1,2}^{(1)} a_{0,1}^{(1) *}, \\
& \dot{a}_{1,-1}^{(1)}=A_{1,1} a_{1,-1}^{(1)}-B_{1,1} a_{0,0}^{(1)} a_{1,-1}^{(1)} \\
& +\left({ }^{0,1} C_{1,-2}^{1,-1}+{ }^{1,1} C_{0,1}^{1,2}\right) a_{0,1}^{(1)} a_{1,-2}^{(1)}+{ }^{0,1} C_{0,2}^{0,3} a_{0,-2}^{(1)} a_{1,1}^{(1)} \\
& +\left({ }^{1,0} C_{0,1}^{1,-1}-{ }^{0,1} C_{1,0}^{1,1}\right) a_{1,0}^{(1)} a_{0,1}^{(1) *}-\left({ }^{1,1} C_{-1,2}^{0,3}+{ }^{1,1} C_{0,-3}^{1,-2}\right) a_{1,2}^{(1)} a_{0,3}^{(1) *} \text {, } \\
& \dot{a}_{0,3}^{(1)}=A_{0,3} a_{0,3}^{(1)}-B_{0,3} a_{0,0}^{(1)} a_{0,3}^{(1)} \\
& +\left({ }^{0,1} C_{0,2}^{0,3}+{ }^{0,2} C_{0,1}^{0,3}\right) a_{0,1}^{(1)} a_{0,2}^{(1)}+\left({ }^{0,3} C_{1,-1}^{1,2}-{ }^{1,1} C_{0,-3}^{1,-2}\right) a_{1,1}^{(1)} a_{1,-2}^{(1) *} \\
& +\left({ }^{1,1} C_{0,-3}^{1,-2}-{ }^{0,3} C_{1,-1}^{1,2}\right) a_{1,-1}^{(1) *} a_{1,2}^{(1)} \text {, } \\
& \dot{a}_{1,2}^{(1)}=A_{1,2} a_{1,2}^{(1)}-B_{1,2} a_{0,0}^{(1)} a_{1,2}^{(1)}+\left({ }^{1,1} C_{0,1}^{1,2}-{ }^{0,1} C_{1,1}^{1,2}\right) a_{0,1}^{(1)} a_{1,1}^{(1)} \\
& +\left({ }^{0,2} C_{1,0}^{1,2}-{ }^{1,0} C_{0,2}^{1,-2}\right) a_{1,0}^{(1)} a_{0,2}^{(1)}+\left({ }^{1,1} C_{-1,2}^{0,3}+{ }^{0,3} C_{1,-1}^{1,2}\right) a_{0,3}^{(1)} a_{1,-1}^{(1)} \text {, }
\end{aligned}
$$




$\begin{array}{lccrl}n_{x} & n_{z} & A_{n_{x}, n_{z}}^{\prime} & A_{n_{x}, n_{z}}^{\prime \prime} & B_{n_{x}, n_{z}} \\ 0 & 0 & 0 & 10.7603 & 0 \\ 0 & 1 & 0.1281 & 9.8256 & 0.0386 \\ 0 & 2 & -0.0503 & 21.8841 & -0.0074 \\ 1 & 0 & 0 & 9.9411 & 0 \\ 1 & 1 & 0.0141 & 14.9003 & 0.0006 \\ 0 & 3 & -0.0562 & 27.9672 & -0.0226 \\ 1 & 2 & 0.2430 & 18.3647 & 0.0876\end{array}$

TABLE 2. Coefficients for the system (3.7).

$$
\begin{aligned}
\dot{a}_{1,-2}^{(1)}= & A_{1,2} a_{1,-2}^{(1)}-B_{1,2} a_{0,0}^{(1)} a_{1,-2}^{(1)}+\left({ }^{0,2} C_{1,0}^{1,2}-{ }^{1,0} C_{0,2}^{1,-2}\right) a_{1,0}^{(1)} a_{0,2}^{(1) *} \\
& -\left({ }^{1,1} C_{-1,2}^{0,3}+{ }^{0,3} C_{1,-1}^{1,2}\right) a_{1,1}^{(1)} a_{0,3}^{(1) *}+\left({ }^{0,1} C_{1,1}^{1,2}-{ }^{1,1} C_{0,1}^{1,2}\right) a_{1,-1}^{(1)} a_{0,1}^{(1) *},
\end{aligned}
$$

where the $A_{n_{x}, n_{z}}$ may be partitioned as

$$
A_{n_{x}, n_{z}} \stackrel{\text { def }}{=} A_{n_{x}, n_{z}}^{\prime}-A_{n_{x}, n_{z}}^{\prime \prime} / R e,
$$

with the $A_{n_{x}, n_{z}}^{\prime}, A_{n_{x}, n_{z}}^{\prime \prime}$ and $B_{n_{x}, n_{z}}$ given in table 2, and the remaining nonlinear terms as follows:

$$
\begin{aligned}
& { }^{0,1} C_{0,1}^{0,2}=0.0647, \quad{ }^{0,1} C_{1,-1}^{1,0}=0.0120, \quad{ }^{0,1} C_{1,0}^{1,1}=0.1833, \quad{ }^{0,1} C_{1,-2}^{1,-1}=0.0445, \\
& { }^{0,1} C_{1,1}^{1,2}=0.1199, \quad{ }^{0,1} C_{0,-3}^{0,-2}=0.0002, \quad{ }^{0,1} C_{0,2}^{0,3}=0.0006, \quad{ }^{0,2} C_{1,-2}^{1,0}=0.0074, \\
& { }^{0,2} C_{1,0}^{1,2}=0.2374, \quad{ }^{0,2} C_{1,-1}^{1,1}=0.0595, \quad{ }^{0,2} C_{0,1}^{0,3}=0.1517, \quad{ }^{1,0} C_{0,1}^{1,-1}=0.0828, \\
& { }^{1,0} C_{0,2}^{1,-2}=0.0083, \quad{ }^{1,1} C_{0,-3}^{1,-2}=0.2413, \quad{ }^{1,1} C_{-1,2}^{0,3}=0.1171, \\
& { }^{1,1} C_{0,1}^{1,2}=0.1723, \quad{ }^{0,3} C_{1,-1}^{1,2}=0.3468 .
\end{aligned}
$$

We refer to this as the 9-coupled-mode model.

The boxes in $(3.7 a-i)$ denote the dominant nonlinear terms, determined as follows. We approximate the average magnitude of a product of amplitudes $a_{n_{x}, n_{z}}^{(n)} a_{m_{x}, m_{z}}^{(m)}$ as $\left\langle\left|a_{n_{x}, n_{z}}^{(n)} a_{m_{x}, m_{z}}^{(m)}\right|\right\rangle \approx\left\langle\left|\hat{a}_{n_{x}, n_{z}}^{(n)}\right|\right\rangle\left\langle\left|\hat{a}_{m_{x}, m_{z}}^{(m)}\right|\right\rangle$, and the average magnitude of an amplitude $a_{n_{x}, n_{z}}^{(n)}$ as $\left\langle\left|a_{n_{x}, n_{z}}^{(n)}\right|\right\rangle \approx \sqrt{\left\langle\left|a_{n_{x}, n_{z}}^{(n)}\right|^{2}\right\rangle}=\sqrt{\lambda_{n_{x} n_{z}}^{(n)}}$. Then, for example, the first two nonlinear terms in the $\dot{a}_{0,1}^{(1)}$ equation have approximate average values

$$
\begin{gathered}
\left\langle\left|B_{0,1} a_{0,0}^{(1)} a_{0,1}^{(1)}\right|\right\rangle=\left|B_{0,1}\right|\left\langle\left|a_{0,0}^{(1)}\right|\left|a_{0,1}^{(1)}\right|\right\rangle \approx\left|B_{0,1}\right|\left\langle\left|\hat{a}_{0,0}^{(1)}\right|\right\rangle\left\langle\left|\hat{a}_{0,1}^{(1)}\right|\right\rangle \approx\left|B_{0,1}\right| \sqrt{\lambda_{0,0}^{(1)}} \sqrt{\lambda_{0,1}^{(1)}}=0.0721, \\
\left\langle\left.\right|^{0,1} C_{0,1}^{0,2} a_{0,2}^{(1)} a_{0,1}^{(1) *} \mid\right\rangle=\left|{ }^{0,1} C_{0,1}^{0,2}\right|\left\langle\left|a_{0,2}^{(1)}\right|\left|a_{0,1}^{(1)}\right|\right\rangle \approx\left|{ }^{0,1} C_{0,1}^{0,2}\right| \sqrt{\lambda_{0,2}^{(1)}} \sqrt{\lambda_{0,1}^{(1)}}=0.0133 .
\end{gathered}
$$

The next largest terms in this sense (those involving $a_{1,1}^{(1)} a_{1,0}^{(1) *}$ and $a_{1,0}^{(1)} a_{1,-1}^{(1) *}$ ) each have approximate average magnitude of 0.003 , and other terms have an even smaller contribution. Since the contribution from the first two terms is, on average, higher than the other terms, we designate them as dominant. Similar considerations apply to the other equations.

While admittedly crude, such approximations allow us to identify the dominant nonlinear interactions among the modes, giving insight into the physics of how turbulence is sustained. We notice, for example, that the first nonlinear term in the 
$\dot{a}_{0,0}^{(1)}$ equation $(3.7 a)$ is the dominant one for the dynamics of the $(1,0,0)$ mode. Since $B_{0,1}>0$, this represents a net flow of energy from the $(1,0,1)$ mode to the $(1,0,0)$ mode. Next, the first two nonlinear terms in the $\dot{a}_{0,1}^{(1)}$ equation $(3.7 b)$ are the dominant ones for the dynamics of the $(1,0,1)$ mode. The first term represents the net flow of energy from the $(1,0,1)$ mode to the $(1,0,0)$ mode, as just mentioned. The second term represents the nonlinear interaction between the $(1,0,1)$ and the $(1,0,2)$ modes; for turbulence to be sustained despite the significant loss of energy to the $(1,0,0)$ mode, this term must give a net flow of energy into the $(1,0,1)$ mode. The dominant nonlinear term in the $\dot{a}_{0,2}^{(1)}$ equation is from the self-interaction of the $(1,0,1)$ mode. The dominant terms in the other amplitude equations almost all involve interactions with the $(1,0,0)$ or $(1,0,1)$ modes. This suggests that the 'core' of the turbulence involves feedback among the $(1,0,0),(1,0,1)$, and $(1,0,2)$ modes, with the former two being most crucial to the dynamics of the other modes.

The nine modes included in this model capture $95.9 \%$ of the kinetic energy on average, and the equations describe a 17-dimensional dynamical system (recall that all but the $n_{x}=n_{z}=0$ modal amplitude coefficients are complex, and that for those triads with two non-zero Fourier wavenumbers, two independent coefficients must be allowed). The laminar $\left(a_{n_{x}, n_{z}}^{(1)}=0\right)$ state for this model (without modelling losses as in $\S 3.3$ ) is unstable at sufficiently large Reynolds numbers due to the linear coefficients of the $(1,0,1),(1,1, \pm 1)$ and $(1,1, \pm 2)$ modes: an unphysical result that motivates the work of $\S 4$.

\subsubsection{Preliminary comments on choice of modal groups}

Since the first four modes of table 1 capture $94.7 \%$ of the average turbulent kinetic energy, it seems reasonable to base a model on these four, adding other modes necessary to retain appropriate Fourier wavenumber interactions. This leads us to consider an 11-dimensional model based on the six modes $((1,0,0),(1,0,1),(1,0,2),(1,1,0)$ and $(1,1, \pm 1))$, the equations for which may be obtained by setting $a_{0,3}^{(1)}=a_{1, \pm 2}^{(1)}=0$ in equations $(3.7 a-f)$ and dropping the equations for the omitted variables. We refer to this as the 6-coupled-mode model.

We also note that the interaction among the $(1,0,0),(1,0,1)$ and $(1,0,2)$ modes, which together capture $93.5 \%$ the most turbulent kinetic energy on average according to table 1 , is determined by the 'energy-conserving' quadratic normal form of the $0: 1: 2$ spatial resonance. While it does not reproduce the turbulent dynamics accurately, it does form an analytically tractable core of larger models such as $(3.7 a-i)$, and we perform a detailed analysis of it elsewhere (Smith, Moehlis \& Holmes 2005).

\subsection{Modelling neglected modes}

Before considering the dynamics of the 6- and 9-coupled-mode models we describe a model for capturing interactions with modes neglected in these truncations. As in $\S 2.3$ we denote the 'true' modal amplitude obtained by projection of the DNS data onto the $\left(n, n_{x}, n_{z}\right)$ mode by $\hat{a}_{n_{x}, n_{z}}^{(n)}$. We follow Podvin \& Lumley (1998) and Podvin (2001) and calculate, for each of these true modal amplitudes, the effect of the neglected modes on the POD mode under consideration, given by

$$
\widehat{T}_{n_{x}, n_{z}}^{(n)}=\sum_{n^{\prime} \neq n} A_{n_{x} n_{y}}^{\left(n^{\prime}, n\right)} \hat{a}_{n_{x}, n_{z}}^{\left(n^{\prime}\right)}+\sum B_{n_{x} n_{z} m_{x} m_{z}}^{(n, m, k)} \hat{a}_{m_{x}, m_{z}}^{(m)} \hat{a}_{n_{x}-m_{x}, n_{z}-m_{z}}^{(k)} .
$$

Here the first sum is over all $n^{\prime}$ such that $\left(n^{\prime}, n_{x}, n_{z}\right)$ is not in the truncation, while the second sum is over all $m, k, m_{x}, m_{z}$ such that $\left(m, m_{x}, m_{z}\right)$ and $\left(k, n_{x}-m_{x}, n_{z}-m_{z}\right)$ are not in the truncation. 
Having calculated this quantity, we determine how well it correlates with the corresponding coefficient $\hat{a}_{n_{x}, n_{z}}^{(n)}$. We define the scalar product between two complexvalued functions of time $f(t)$ and $g(t)$ as

$$
\langle f \mid g\rangle \stackrel{\text { def }}{=} \lim _{t \rightarrow \infty} \int_{0}^{t} f(s) g^{*}(s) \mathrm{d} s,
$$

with the associated norm

$$
\|f\| \stackrel{\text { def }}{=}\langle f \mid f\rangle^{1 / 2} \text {. }
$$

The correlation coefficient between $f(t)$ and $g(t)$ is then defined to be

$$
C(f, g) \stackrel{\text { def }}{=} \frac{\operatorname{Re}[\langle f \mid g\rangle]}{(\langle f \mid f\rangle\langle g \mid g\rangle)^{1 / 2}} .
$$

If $\widehat{T}_{n_{x}, n_{z}}^{(n)}$ and $\hat{a}_{n_{x}, n_{z}}^{(n)}$ are significantly anti-correlated $(-C>0.5$, say), we may employ the linear approximation

$$
\widehat{T}_{n_{x}, n_{z}}^{(n)} \approx-\beta_{n_{x}, n_{z}}^{(n)} \hat{a}_{n_{x}, n_{z}}^{(n)} ; \quad \beta_{n_{x}, n_{z}}^{(n)}>0,
$$

which allows closure of the truncated system by approximating $\widehat{T}_{n_{x}, n_{z}}^{(n)}$ by an additional damping term:

$$
\dot{a}_{n_{x}, n_{z}}^{(n)}=\text { terms from truncation }-\beta_{n_{x}, n_{z}}^{(n)} a_{n_{x}, n_{z}}^{(n)} .
$$

The error induced in (3.13),

$$
\left\|\widehat{T}_{n_{x}, n_{z}}^{(n)}+\beta_{n_{x}, n_{z}}^{(n)} \hat{a}_{n_{x}, n_{z}}^{(n)}\right\|^{2}=\int_{0}^{t}\left(\widehat{T}_{n_{x}, n_{z}}^{(n)}+\beta_{n_{x}, n_{z}}^{(n)} \hat{a}_{n_{x}, n_{z}}^{(n)}\right)\left(\widehat{T}_{n_{x}, n_{z}}^{(n) *}+\beta_{n_{x}, n_{z}}^{(n)} \hat{a}_{n_{x}, n_{z}}^{(n) *}\right) \mathrm{d} t,
$$

can be minimized in a least-squares sense by setting

$$
\beta_{n_{x}, n_{z}}^{(n)} \stackrel{\text { def }}{=}-\frac{\int_{0}^{t}\left(\widehat{T}_{n_{x}, n_{z}}^{(n)} \hat{a}_{n_{x}, n_{z}}^{(n) *}+\hat{a}_{n_{x}, n_{z}}^{(n)} \widehat{T}_{n_{x}, n_{z}}^{(n) *}\right) \mathrm{d} t}{2 \int_{0}^{t}\left|\hat{a}_{n_{x}, n_{z}}^{(n)}\right|^{2} \mathrm{~d} t},
$$

which reveals the optimal damping required for each mode.

Applying this below we find that the correlation coefficients $C\left(\widehat{T}_{n_{x}, n_{z}}^{(n)}, \hat{a}_{n_{x}, n_{z}}^{(n)}\right)$ vary considerably and the approximation (3.13) is thus more convincing for some modes than others. We shall attempt to reduce the effects of this variability by lumping the damping coefficients $\beta_{n_{x}, n_{z}}^{(n)}$ into a single model-specific damping coefficient via the weighted average over all modes contained in the model:

$$
v \stackrel{\text { def }}{=}\left\langle\beta_{n_{x}, n_{z}}^{(n)} /\left(n_{x}^{2}+n_{z}^{2}\right)\right\rangle .
$$

Losses to each mode will then be approximated as

$$
\widehat{T}_{n_{x}, n_{z}}^{(n)} \approx-\alpha v\left(n_{x}^{2}+n_{z}^{2}\right) a_{n_{x}, n_{z}}^{(n)},
$$

where $\alpha=1$ corresponds to a standard spectral eddy viscosity model (cf. Pope 2000, p. 610).

Equation (3.17) implies that $\widehat{T}_{0.0}^{(n)}$ is identically zero, and thus no attempt is made to model the influence of the neglected terms on the $(1,0,0)$ mode. This 'mean flow' mode is problematic since first, for all truncations considered, $\widehat{T}_{0,0}^{(1)}$ oscillates about a mean close to zero, whilst the mean of $a_{0,0}^{(1)}$ hovers around 2.1. It is thus no simple matter to approximate $\widehat{T}_{0,0}^{(1)}$ with a function of the form $-\beta a_{0,0}^{(1)}$. Secondly, plots of $\widehat{T}_{0,0}^{(1)}$ 

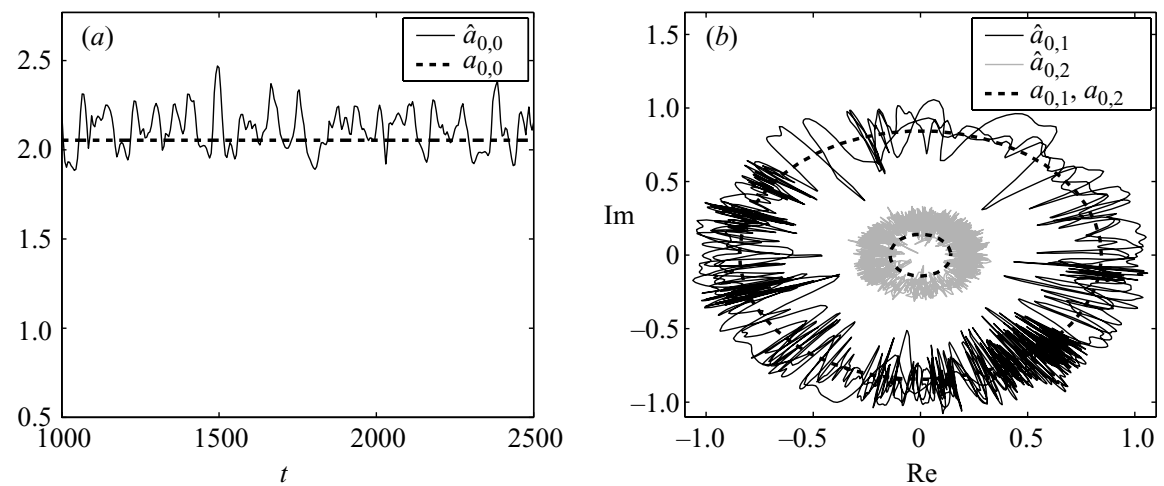

FiguRE 9. Behaviour of the 6-coupled-mode model compared to the DNS projected onto the $(1,0,0),(1,0,1)$ and $(1,0,2)$ modes. Here each of $a_{0,0}, a_{0,1}, a_{0,2}$ are shown as dashed lines, and are intended to represent approximations to the nearest (noisy) $\hat{a}_{n_{x}, n_{z}}$ line.

amply demonstrate the fact that the $a_{0,0}^{(1)}$ mode is often subject to backscatter: energy flows from the neglected modes back to this mode. Indeed, equation (3.7a) indicates that energy flows from the $\left(1, n_{x}, n_{z}\right)$ mode into the $(1,0,0)$ mode whenever $B_{n_{x}, n_{z}}$ is positive, and this is a primary mechanism sustaining non-trivial behaviour identified in Moehlis et al. (2002).

In carrying out the average (3.16) we reduce the (bifurcation) parameters to two: namely, the Reynolds number $R e$ and $\alpha$, an $O(1)$ parameter adjusting the losses to the neglected modes.

\subsection{Dynamical behaviour of the models involving coupled modes}

\subsubsection{The 6-coupled-mode model}

We now consider the dynamics of the 6-coupled-mode model, constructed from a Galerkin projection of $(1.1)$ onto the $(1,0,0),(1,0,1),(1,0,2),(1,1,0),(1,1, \pm 1)$ modes. Here and throughout $\S 3$, all computations are done at $R e=400$. Integration of this 11-dimensional dynamical system, without modelling losses to neglected modes, reveals travelling waves of the form

$$
a_{n_{x}, n_{z}}^{(n)}=r_{n_{x}, n_{z}}^{(n)} \exp \left(\mathrm{i}\left(-\omega n_{z} t+\alpha_{n_{x}, n_{z}}^{(n)}\right)\right),
$$

where reality of $a_{0,0}^{(1)}$ requires that the phase $\alpha_{0,0}^{(1)}$ is necessarily zero. The amplitudes of the $(1,0,0),(1,0,1)$ and $(1,0,2)$ modes are plotted in figure 9 in comparison with the 'true' modal amplitudes obtained by projecting the DNS onto these modes, denoted $\hat{a}_{0, j}$.

Note that $\hat{a}_{0,1}^{(1)}$ and $\hat{a}_{0,2}^{(1)}$ are approximately confined to a 'thickened' torus: each oscillates relatively quickly along a radius and drifts more slowly and chaotically around the circumference. The model fails to reproduce the radial motion, and caricatures the circumferential motion as a simple travelling wave with a relatively low period of 65.9 time units. It is clear from figure 9 that the average of the projected $(1,0,0),(1,0,1)$ and $(1,0,2)$ modal amplitudes is approximated well by the travelling wave model: this is less true of the $(1,1,0)$ and $(1,1, \pm 1)$ modal amplitudes, as indicated by table 3 , where the eigenvalues $\lambda_{n_{x}, n_{z}}^{(n)}$ are compared with the square of the travelling wave amplitudes $\left\|a_{n_{x}, n_{z}}^{(n)}\right\|=r_{n_{x}, n_{z}}^{(n)}$ (recall that the latter are twice the modal energies: $§ 2.3$ ). 


$\begin{array}{lcc}\left(n, n_{x}, n_{y}\right) & \lambda_{n_{x}, n_{z}}^{(n)} & \left(r_{n_{x}, n_{z}}^{(n)}\right)^{2} \\ (1,0,0) & 4.4550 & 4.2171 \\ (1,0, \pm 1) & 0.7821 & 0.7134 \\ (1,0, \pm 2) & 0.0543 & 0.0202 \\ (1, \pm 1,0) & 0.0386 & 0.2791 \\ (1, \pm 1, \pm 1) & 0.0090 & 0.1752\end{array}$

TABLE 3. Mean behaviour of the undamped 6-coupled-mode model.
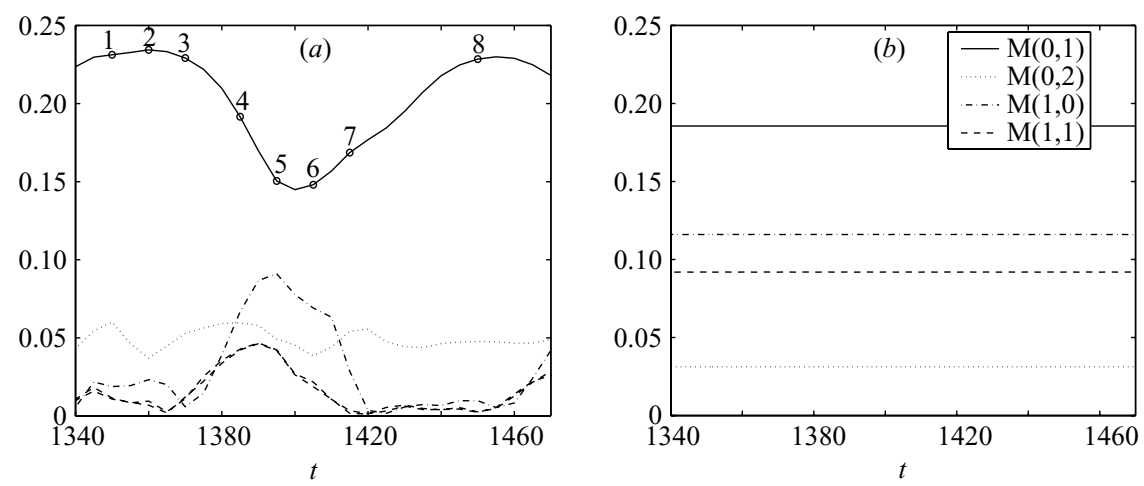

FIGURE 10. RMS modal velocities: from a representative cycle of the DNS ( $a$, computed from (3.19) for $n=1$, and thus slightly different from the figure $2 b$ ) and analogous (constant) quantities for the 6-coupled-mode model $(b)$.

The travelling wave solution is, however, entirely unsatisfactory from a dynamical viewpoint. The RMS modal velocities, calculated from

$$
\mathrm{M}\left(n_{x}, n_{z}\right)=\sqrt{\frac{\sum_{n}\left|a_{n_{x}, n_{z}}^{(n)}\right|^{2}}{L_{x} L_{z}}},
$$

are constant, since the modal amplitudes are constant: see figure $10(b)$. The HKW regeneration cycle is completely absent. Here, and for similar plots in this paper, we present in panel $(a)$ the analogous quantities for the DNS with only those modes present in the low-dimensional model included in the projection, i.e. (3.19) with $a_{n_{x}, n_{z}}^{(n)}$ replaced by $\hat{a}_{n_{x}, n_{z}}^{(n)}$. In no case do we sum over $n$, since only one quantum number is included in the models considered in this paper.

Substituting (3.18) into the Galerkin approximation (2.4) yields

$$
\boldsymbol{u}(\boldsymbol{x}, t)=\sum \frac{r_{n_{x}, n_{z}}^{(n)}}{\sqrt{L_{x} L_{z}}} \exp \left(2 \pi \mathrm{i}\left(\frac{n_{x} x}{L_{x}}+\frac{n_{z}\left(z-\omega L_{z} t /(2 \pi)\right)}{L_{z}}+\frac{\alpha_{n_{x}, n_{z}}^{(n)}}{2 \pi}\right)\right) \boldsymbol{\phi}_{n_{x}, n_{z}}^{(n)}(y),
$$

hence the travelling waves (3.18) represent streak/vortex structures moving in the spanwise direction at speed $\omega L_{z} /(2 \pi)$.

\subsubsection{The 6-coupled-mode model with losses to neglected modes}

We now attempt to improve the model behaviour by including energy transfer to neglected modes, using the ideas of $\S 3.3$. The resulting correlation coefficients and optimal damping values, calculated from (3.9)-(3.15), are presented in table 4. There 


$\begin{array}{rrrcc}n & n_{x} & n_{z} & C\left(\widehat{T}_{n_{x}, n_{z}}^{(n)}, a_{n_{x}, n_{z}}^{(n)}\right) & \beta_{n_{x}, n_{z}}^{(n)} \\ 1 & 0 & 1 & -0.2650 & 0.0024 \\ 1 & 0 & 2 & -0.8870 & 0.1294 \\ 1 & 1 & 0 & -0.3086 & 0.0229 \\ 1 & 1 & 1 & -0.5150 & 0.1039 \\ 1 & 1 & -1 & -0.5136 & 0.1038\end{array}$

TABLE 4. Correlation coefficients for the 6-coupled-mode model.
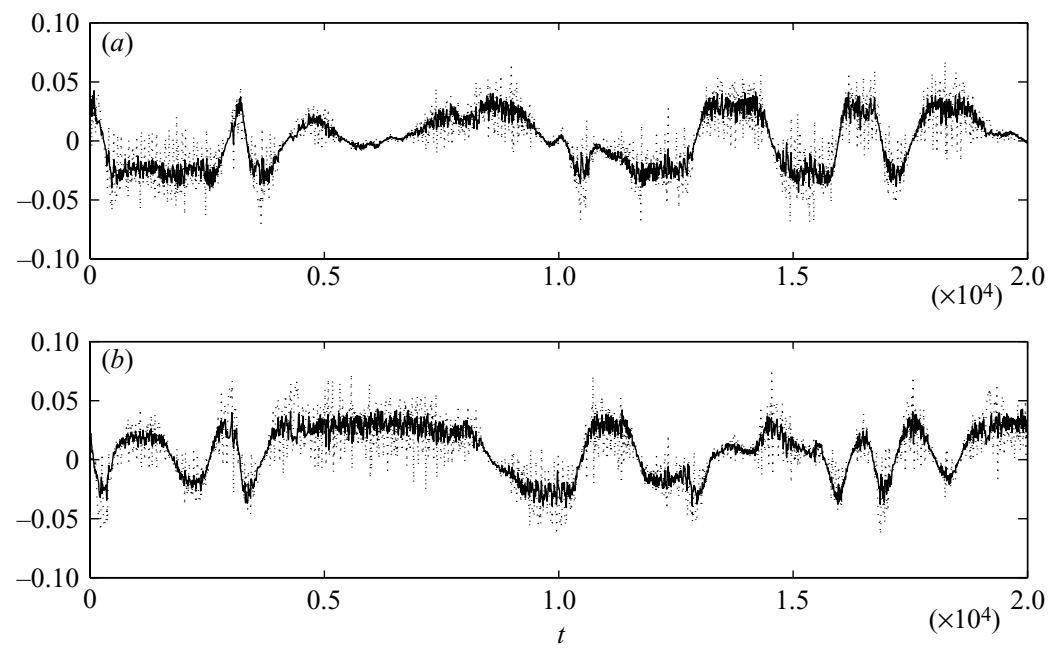

FIGURE 11. For the 6-coupled-mode model: $(a)$ plot of $\operatorname{Re}\left(-\beta_{0,2}^{(1)} \hat{a}_{0,2}^{(1)}\right)$ (solid) and $\operatorname{Re}\left(\widehat{T}_{0,2}^{(1)}\right)$ (dashed) against time, $t ;(b)$ plot of $\operatorname{Im}\left(-\beta_{0,2}^{(1)} \hat{a}_{0,2}^{(1)}\right)$ (solid) and $\operatorname{Im}\left(\widehat{T}_{0,2}^{(1)}\right)$ (dashed) against time, $t$. This reflects the high correlation coefficient of -0.8870 of table 4 .

is significant variation in $C\left(\widehat{T}_{n_{x}, n_{z}}^{(n)}, a_{n_{x}, n_{z}}^{(n)}\right)$, indicating that the quality of the linear damping approximation varies widely. To illustrate, we exhibit the best- and worstapproximated modes in figures 11 and 12 , respectively. Averaging this variability via (3.16)-(3.17), we obtain the eddy viscosity $v=0.0333$.

We now adjust the $O(1)$ parameter $\alpha$ to obtain the best fit between the behaviour of the model and the DNS by computing a bifurcation diagram using the program AUTO (Doedel et al. 1997), shown schematically in figure 13. This shows how existence and stability of solutions depends on $\alpha$. As $\alpha$ increases from zero, we successively encounter the previously described travelling waves (TW), modulated travelling waves (MW), and two different types of standing waves confined to a rotated version of the real subspace: $\left(\mathrm{SW}_{1}\right.$ and $\left.\mathrm{SW}_{2}\right)$. Schematic diagrams of the behaviour of the $(1,0,1)$ and $(1,0,2)$ modal amplitudes for these solutions are shown at the bottom of figure 13; at the left are the travelling wave solutions shown in figure 9. All solutions with the exception of MW are stable over some interval of $\alpha$. We draw the reader's attention to the branch of fixed points at the right, which are produced in a pitchfork bifurcation from the laminar state at a higher value of $\alpha(\sim 1.4)$; indeed, as we noted in $\S 3.2$ the laminar state of this model is unstable for Reynolds numbers in the vicinity of $R e=400$. 

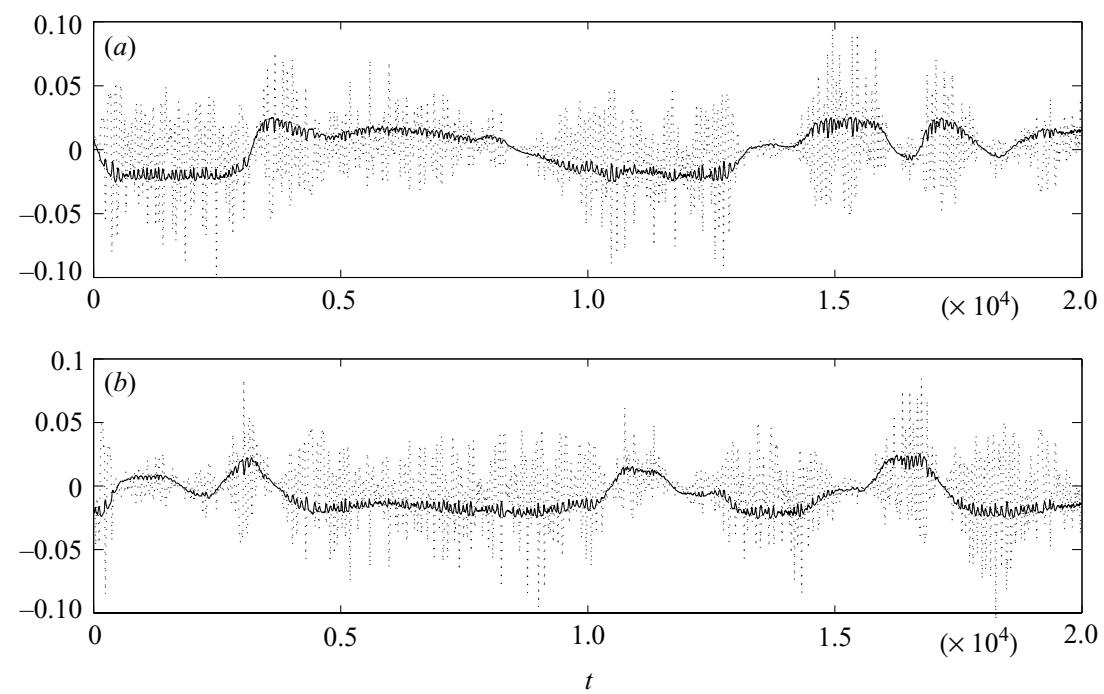

FIGURE 12. For the 6-coupled-mode model: $(a)$ plot of $\operatorname{Re}\left(-10 \beta_{0,1}^{(1)} \hat{a}_{0,1}^{(1)}\right)$ (solid) and $\operatorname{Re}\left(\widehat{T}_{0,1}^{(1)}\right)$ (dashed) against time, $t ;(b)$ plot of $\operatorname{Im}\left(-10 \beta_{0,1}^{(1)} \hat{a}_{0,1}^{(1)}\right)$ (solid) and $\operatorname{Im}\left(\widehat{T}_{0,1}^{(1)}\right)$ (dashed) against time, $t$. The optimal least-squares approximation in this case, with $\beta=-0.0024$ is essentially flat; we have multiplied this damping factor by 10 to show that the linear approximation (3.13) captures some of the low-frequency part of the noisy $\widehat{T}_{0,1}^{(1)}$ term. This reflects the low correlation coefficient of -0.2650 of table 4 .

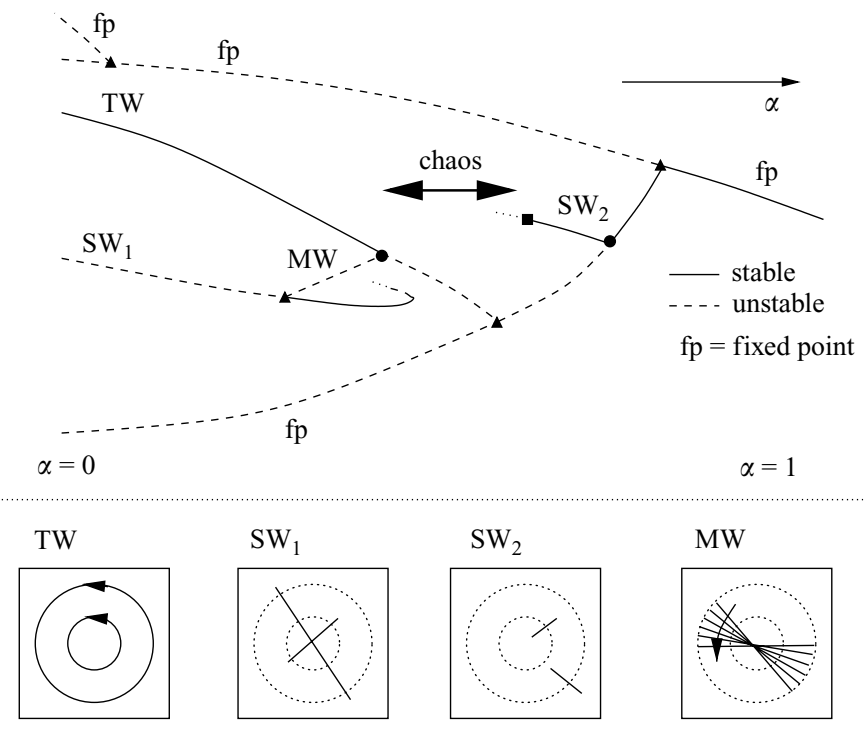

FIGURE 13. Schematic bifurcation diagram with respect to $\alpha$ for the 6-coupled-mode model.

The stable $\mathrm{SW}_{2}$ solution, for which the $(1,0,1)$ and $(1,0,2)$ modal amplitudes oscillate along radii, captures the appropriate DNS dynamics, its period of 96 nondimensional time units for $\alpha=0.8$ comparing favourably with the recurrence period for streak breakdown of 80-100 non-dimensional time units from our and other DNS simulations: see figure 14 and cf. figure 9. However, while the amplitudes of the 

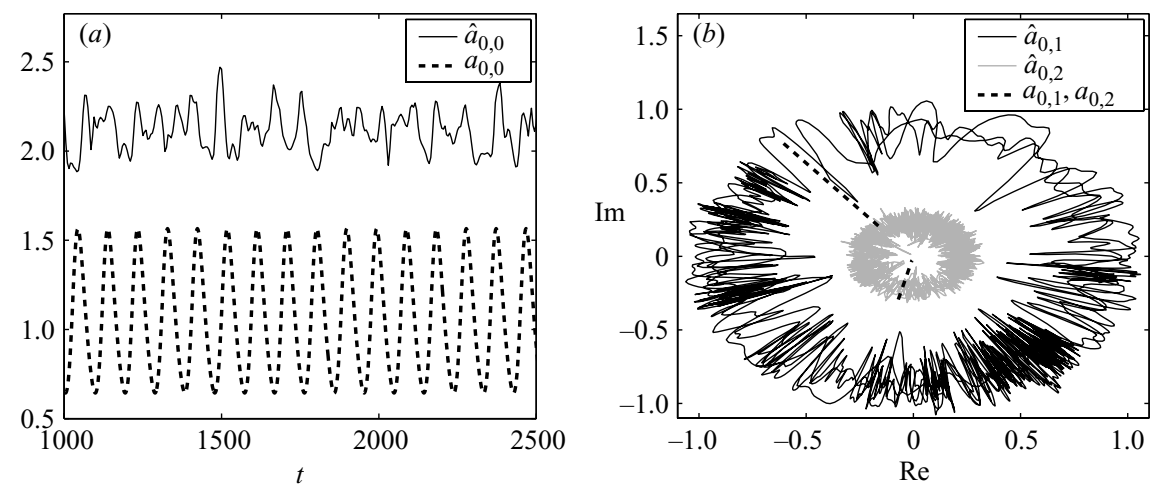

FIgURE 14. Behaviour of the 6-coupled-mode model with $\alpha=0.8$ compared to the DNS projected onto the $(a)(1,0,0)$ and $(b)(1,0,1),(1,0,2)$ modes.

(a)

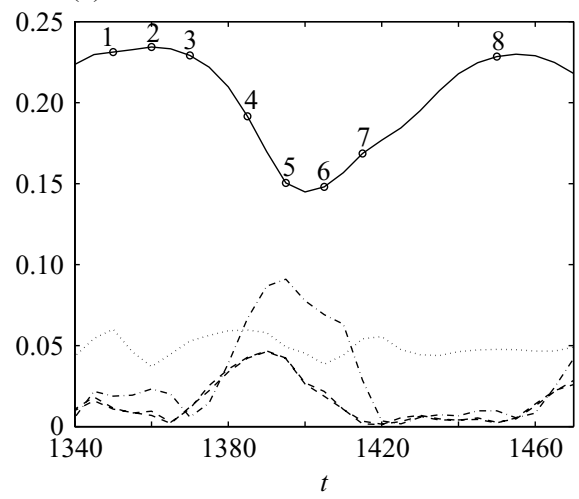

(b)

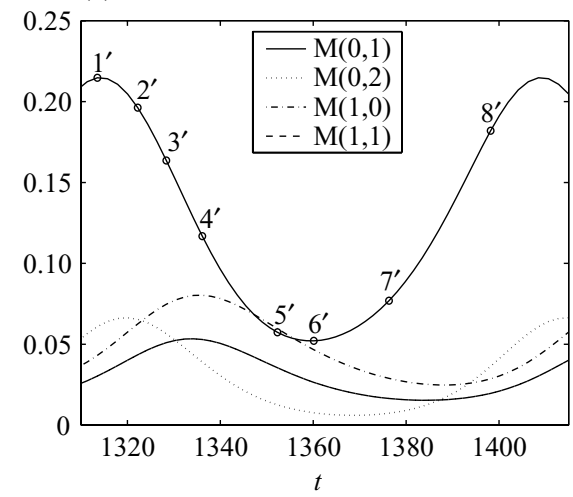

FIGURE 15. RMS modal velocities: $(a)$ from a representative cycle of the DNS (repeated from figure 10$)$ and $(b)$ for one period from the 6-coupled-mode model, including modelling of losses to neglected modes with $\alpha=0.8$.

complex modes are reasonable, that of the $(1,0,0)$ mode in the model is significantly lower than in the projected DNS.

The RMS modal velocities for the model, calculated from (3.19), are presented in figure 15(b), for comparison with analogous DNS quantities, re-plotted in figure 15( $a)$ (cf. figure 2). The cyclic behaviour is essentially reproduced: in both cases $\mathrm{M}(1,0)$ and $\mathrm{M}(1,1)$ are in phase, although in the model they are $1 / 4$ cycle out of phase with $\mathrm{M}(0,1)$ in contrast to $1 / 2$ cycle in the DNS, and their minimum amplitudes are higher than in the DNS.

In figures 16 and 17 we show reconstructed model velocity fields at the time instants $1^{\prime}-8^{\prime}$ marked on figure 15(b) (analogous to 1-8 on $(a)$ ), for comparison with analogous quantities from the DNS. Panels $(a)$ of these figures repeat data from figures 3 and 4, but projected only onto the 6 modes present in the model (so the majority of the small scales is removed, leaving essential large-scale structures). Figure 16 shows that the solution provides reasonable reconstructions of the streak breakdown process in the $(x, z)$ mid-plane, although the model's streaks do not straighten as much at points 1 and $7-8$ as those of the DNS, due to the phase and amplitude differences noted above, and figure 17 indicates that the agreement between the streamwise velocity contours 


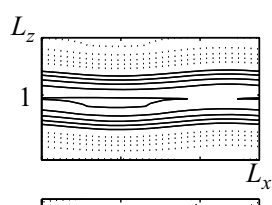

(a)

(b)
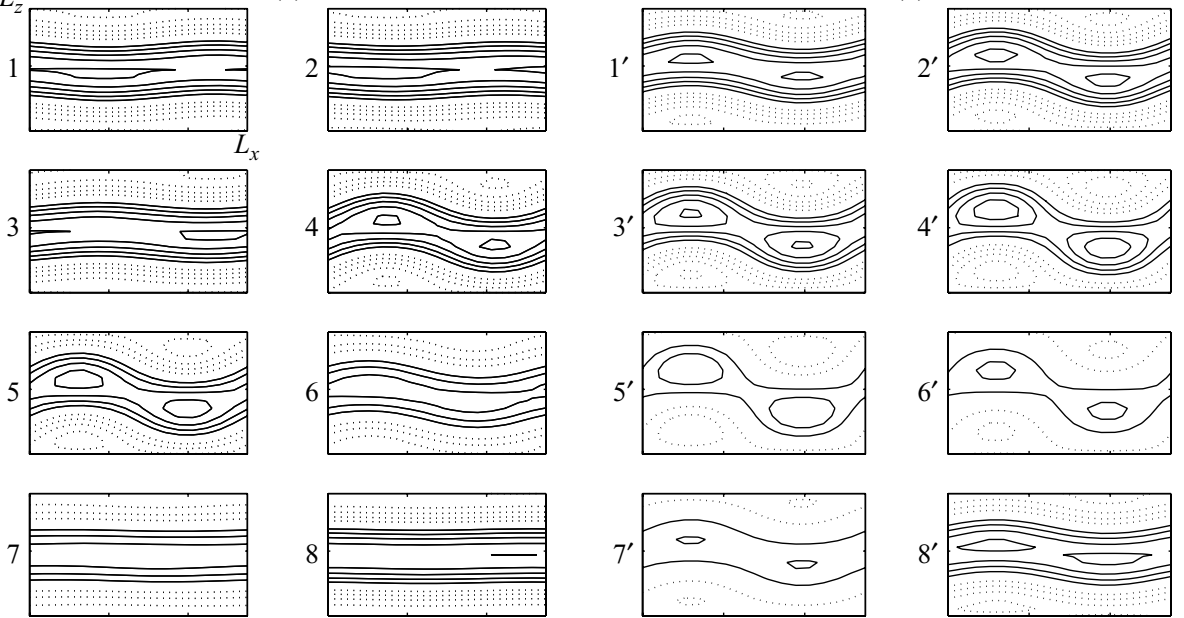

FIGURE 16. The streak-breakdown process viewed in the $(x, z)$-plane lying between the two plates in PCF: DNS projected onto the 6 modes present in the model $(a)$ and computed from one period of the 6-coupled-mode model with $\alpha=0.8(b)$.

(a)

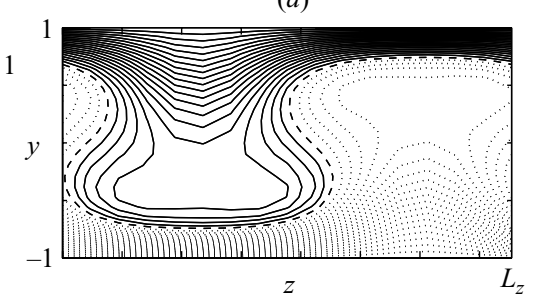

5

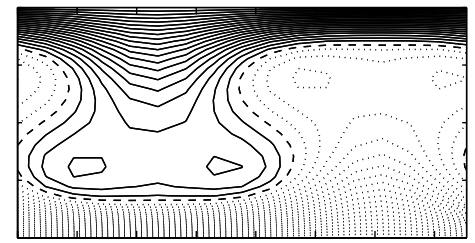

(b)

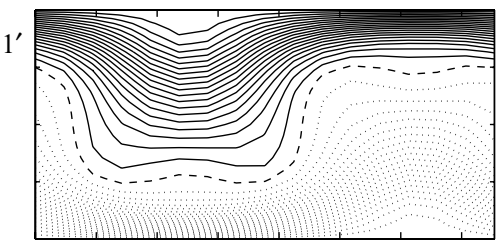

$5^{\prime}$

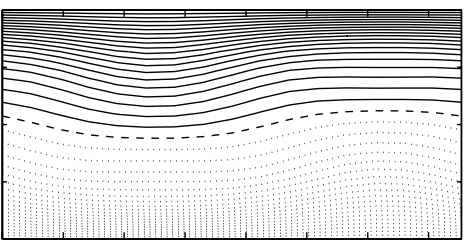

FIGURE 17. Streamwise velocities in the $(y, z)$-plane calculated from the streamwise-invariant modes and the laminar state: projection of the DNS onto the 6 modes present in the model (a) and computed from the 6-coupled-mode model with $\alpha=0.8(b)$.

(as calculated from the streamwise-invariant modes) is somewhat less striking. This is largely due to the low amplitude of the $(1,0,0)$ mode: subtracting the laminar solution to obtain figure 18 , we observe much more striking similarity between the DNS and the low-dimensional model.

Finally we compute turbulent statistics for the model by reconstructing the velocity field over one period of the limit cycle and calculating mean velocity fluctuations $\left(u^{\prime}, v^{\prime}, w^{\prime}\right)$. Figure 19 compares these with analogous DNS quantities. We note good qualitative agreement for streamwise and spanwise RMS fluctuations $\left(\sqrt{\left\langle u^{\prime 2}\right\rangle}\right.$ and $\sqrt{\left\langle v^{\prime 2}\right\rangle}$, respectively) and also for average Reynolds stress $\left(\left\langle u^{\prime} v^{\prime}\right\rangle\right)$. (The agreement between instantaneous Reynolds stress from the DNS and model is best when streaks are prominent; the agreement is poorer during the streak breakdown process, because 

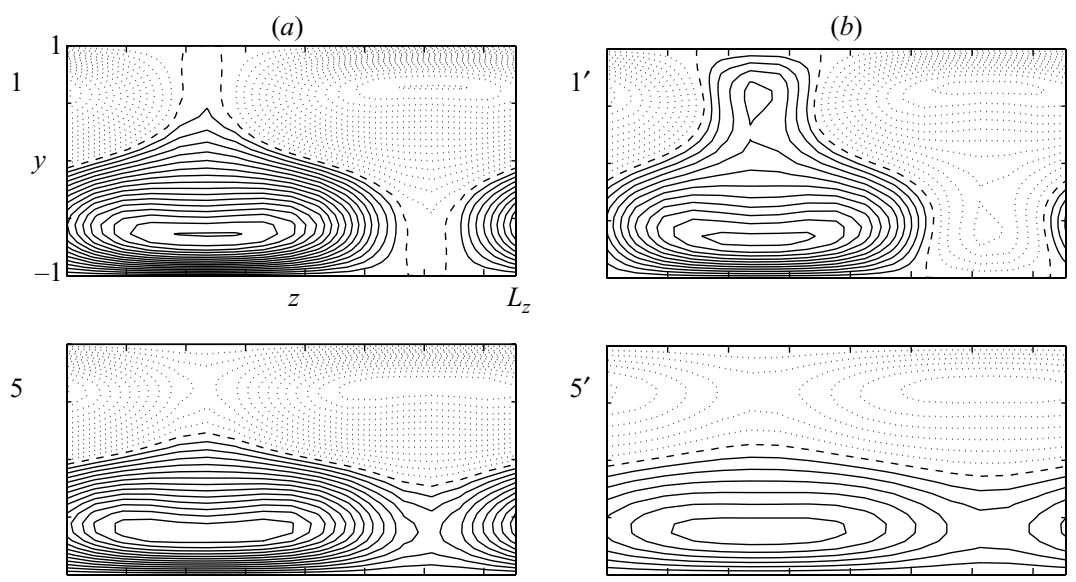

FiguRE 18. Streamwise velocities in the $(y, z)$-plane calculated from the streamwise-invariant modes only (excluding the laminar state): projection of the DNS onto the 6 modes present in the model $(a)$ and computed from the 6-coupled-mode model with $\alpha=0.8(b)$.
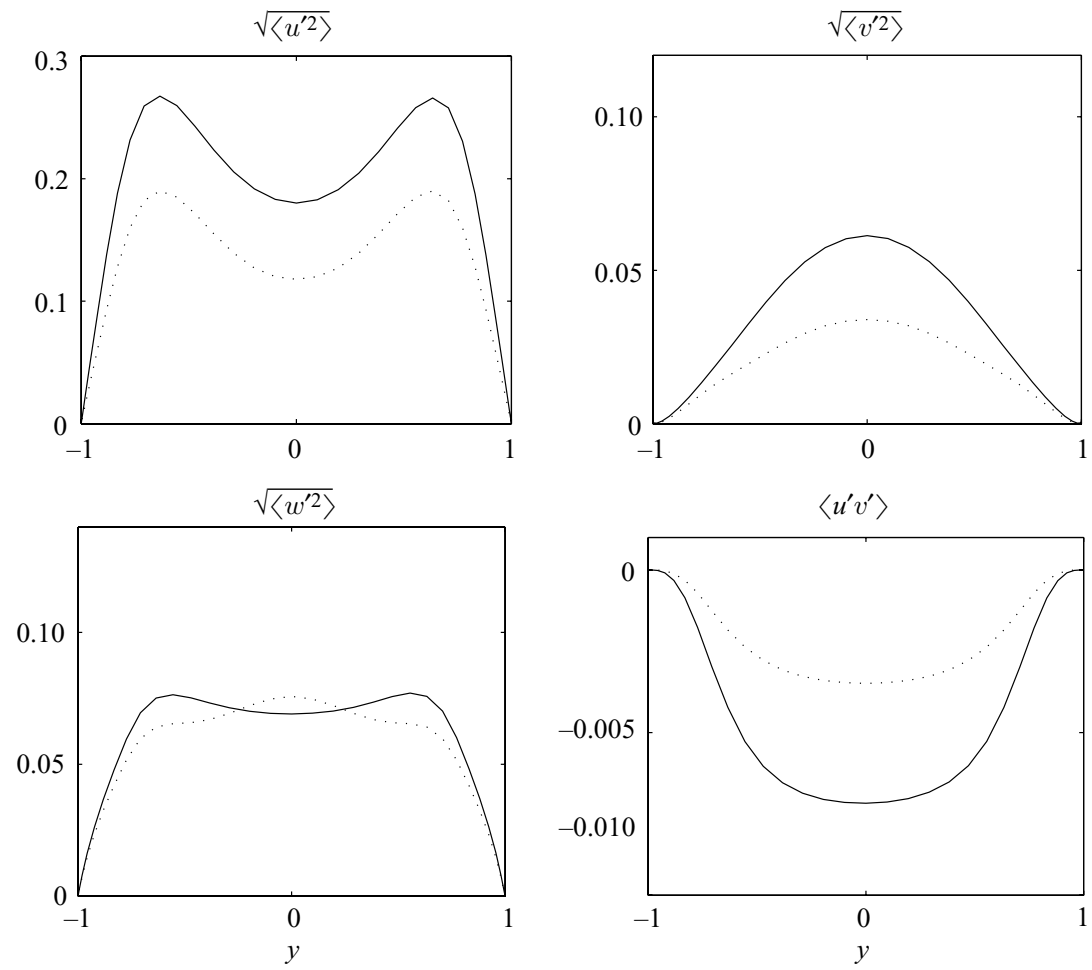

FIGURE 19. Performance of the 6-coupled-mode model with $\alpha=0.8$ measured by turbulent statistics with DNS (solid) and model (dotted) shown.

the model excludes modes needed to resolve the small structures and mixing processes.) The qualitative agreement is less striking in the wall-normal direction $\left(\sqrt{\left\langle w^{\prime 2}\right\rangle}\right)$, with a peak dominating the central portion between the two flat plates rather than a trough, although quantitative agreement is better. 


$\begin{array}{lll}\left(n, n_{x}, n_{y}\right) & \lambda_{n_{x}, n_{z}}^{(n)} & \left(r_{n_{x}, n_{z}}^{(n)}\right)^{2} \\ (1,0,0) & 4.4550 & 4.0487 \\ (1,0, \pm 1) & 0.7821 & 0.7047 \\ (1,0, \pm 2) & 0.0543 & 0.0318 \\ (1, \pm 1,0) & 0.0386 & 0.2553 \\ (1, \pm 1,1) & 0.0090 & 0.1140 \\ (1, \pm 1,-1) & 0.0090 & 0.2345 \\ (1,0, \pm 3) & 0.0543 & 0.0006\end{array}$

TABLE 5. Mean behaviour of the naive ' 6 -coupled-mode $+(1,0, \pm 3)$ ' model.

\subsubsection{The 9-coupled-mode model}

Adding the $(1,0,3)$ mode to the 6-coupled-mode model considered above produces similar dynamic behaviour: in particular, the travelling waves (3.18) persist and the energy budget of the leading four model modes remains reasonable, as table 5 indicates. Moreover, addition of damping to this model to represent losses to neglected modes produces a periodic orbit with behaviour similar to that of the damped 6coupled-mode model of $\S 3.3$.

However, if we add the $(1,1, \pm 2)$ modes to obtain the 'full' set of equations (3.7) of the 9-coupled-mode model, the dynamic behaviour changes markedly: instead of travelling wave solutions we now observe chaos in which the amplitude coefficients explore an appropriate region of phase space, but the dynamics do not approximate the thickened tori of figure 9. Nor did it seem possible to improve the behaviour of this model by addition of eddy viscosity terms. Accordingly, we shall discuss this model no further here, although we shall see in $\S 4$ that a version of it using uncoupled modes produces much more relevant behaviour.

\section{Low-dimensional models using uncoupled modes}

Commenting on the wall layer model of Aubry et al. (1988) which uses only streamwise-invariant modes $\left(n_{x}=0\right.$ in the present notation) presented in Holmes (1990), Moffatt (1990) pointed out that, based on fundamental considerations, all disturbances should eventually decay unless streamwise variations are included. Indeed, for streamwise-invariant flow, for which the convective derivative reduces to

$$
\mathrm{D} / \mathrm{D} t=\partial / \partial t+u_{2} \partial / \partial y+u_{3} \partial / \partial z
$$

the $x$-component of the Navier-Stokes equation is

$$
\frac{\mathrm{D}}{\mathrm{D} t}\left(U+u_{1}\right)=\frac{1}{R e}\left(\frac{\partial^{2}}{\partial y^{2}}+\frac{\partial^{2}}{\partial z^{2}}\right)\left(U+u_{1}\right),
$$

where $U$ denotes the laminar profile, entirely in the $x$-direction, and $u_{1,2,3}$ are the fluctuations. (Note that $u_{2}$ and $u_{3}$ influence the dynamics of $u_{1}$, but $u_{1}$ does not influence the dynamics of $u_{2}$ and $u_{3}$.) Furthermore, it can be shown that

$$
\frac{\mathrm{d}}{\mathrm{d} t} \iint\left(u_{2}^{2}+u_{3}^{2}\right) \mathrm{d} y \mathrm{~d} z=-2 v\left\{\iint \omega_{x}^{2} \mathrm{~d} y \mathrm{~d} z\right\},
$$

where $\omega_{x}$ is the $x$-component of the vorticity, showing that the energy in the crossstream components of the velocity must decay to zero. In the long-time limit then, in (4.2), D/D $t \rightarrow \partial / \partial t$, giving a simple diffusion equation for $u_{1}$. Hence at large time 
$u_{1}$ tends to a constant value, the only possible solution being $u_{1}=0$, and although the streamwise velocity may experience transient growth due the cross-stream flow, it must also eventually vanish.

As pointed out in an addendum to Holmes (1990), in more detail in Berkooz et al. (1991), and directly after equation (2.5) above, the vector-valued POD eigenfunctions impose artificial coupling between streamwise and cross-stream components in each mode, fixing their relative magnitudes and phases. In particular, this implies that the inner product in the second term in the linear coefficients $\hat{A}_{n_{x}, n_{z}}^{(n, k)}$ of (3.2) with $n_{x}=0$ is non-zero, and, in fact, positive for $n=k=1$, providing the energy source in our coupled models. Hence the constraint imposed by projection onto streamwise-invariant modes that (correctly) represents typical behaviour with streamwise fluctuations present, imposes coupling that can paradoxically maintain streamwise velocity fluctuations in models lacking $n_{x} \neq 0$ modes, as in Aubry et al. (1988) and Moehlis et al. (2002).

To remove this constraint and restore the correct physics, we must allow the streamwise and cross-stream velocity components of streamwise-invariant modes to evolve separately. The solution proposed here, which extends the start made in Berkooz et al. (1991), cannot of course restore the infinitely many degrees of freedom on which the Navier-Stokes equations play, but we will show that it does restore sufficient freedom to remove a major problem inherent in very low-dimensional truncations with coupled modes: instability of the laminar state. It also produces ODEs with a correctly nonnormal linear part, as described in $\S 4.4$. That it prevents sustained turbulence in the absence of $n_{x} \neq 0$ modes was already shown in Berkooz et al. (1991); also see $\S 4.3 .1$.

\subsection{Uncoupled basis functions}

Following the suggestion of Waleffe $(1995 \mathrm{~b})$, we construct a pair of uncoupled basis functions from each empirical eigenfunction by decomposing it into mutually orthogonal components:

$$
\boldsymbol{\Phi}_{n_{x}, n_{z}}^{(n)}(\boldsymbol{x})=\boldsymbol{\Phi}_{n_{x}, n_{z}}^{(n)[1]}(\boldsymbol{x})+\boldsymbol{\Phi}_{n_{x}, n_{z}}^{(n)[2]}(\boldsymbol{x}),
$$

where

$$
\boldsymbol{\Phi}_{n_{x}, n_{z}}^{(n)[1]}(\boldsymbol{x}) \stackrel{\text { def }}{=} P_{n_{x}, n_{z}} \boldsymbol{\Phi}_{n_{x}, n_{z}}^{(n)}(\boldsymbol{x}), \quad \boldsymbol{\Phi}_{n_{x}, n_{z}}^{(n)[2]}(\boldsymbol{x}) \stackrel{\text { def }}{=}\left(I-P_{n_{x}, n_{z}}\right) \boldsymbol{\Phi}_{n_{x}, n_{z}}^{(n)}(\boldsymbol{x})
$$

and the projection matrix is defined by

$$
P_{n_{x}, n_{z}} \stackrel{\text { def }}{=} p p^{T} /\left(p^{T} p\right) \text { with } p \stackrel{\text { def }}{=}\left[-2 \pi n_{z} / L_{z}, 0,2 \pi n_{x} / L_{x}\right]^{T} .
$$

Here $\boldsymbol{\Phi}_{n_{x}, n_{z}}^{(n)[1]}(\boldsymbol{x})$ and $\boldsymbol{\Phi}_{n_{x}, n_{z}}^{(n)[2]}(\boldsymbol{x})$ are of the form (2.3) with

$$
\begin{gathered}
\boldsymbol{\phi}_{n_{x}, n_{z}}^{(n)[1]}(y)=\left(\begin{array}{c}
a \boldsymbol{\phi}_{1, n_{x}, n_{z}}^{(n)}(y)-b \boldsymbol{\phi}_{3, n_{x}, n_{z}}^{(n)}(y) \\
0 \\
-b \boldsymbol{\phi}_{1, n_{x}, n_{z}}^{(n)}(y)+c \boldsymbol{\phi}_{3, n_{x}, n_{z}}^{(n)}(y)
\end{array}\right), \\
\boldsymbol{\phi}_{n_{x}, n_{z}}^{(n)[2]}(y)=\left(\begin{array}{c}
(1-a) \boldsymbol{\phi}_{1, n_{x}, n_{z}}^{(n)}(y)+b \boldsymbol{\phi}_{3, n_{x}, n_{z}}^{(n)}(y) \\
\boldsymbol{\phi}_{2, n_{x}, n_{z}}^{(n)}(y) \\
b \boldsymbol{\phi}_{1, n_{x}, n_{z}}^{(n)}(y)+(1-c) \boldsymbol{\phi}_{3, n_{x}, n_{z}}^{(n)}(y)
\end{array}\right),
\end{gathered}
$$

respectively, appearing in the place of $\boldsymbol{\phi}_{n_{x}, n_{z}}^{(n)}(y)$, and where

$$
a \stackrel{\text { def }}{=} \frac{n_{z}^{2} / L_{z}^{2}}{n_{x}^{2} / L_{x}^{2}+n_{z}^{2} / L_{z}^{2}}, \quad b \stackrel{\text { def }}{=} \frac{n_{x} n_{z} /\left(L_{x} L_{z}\right)}{n_{x}^{2} / L_{x}^{2}+n_{z}^{2} / L_{z}^{2}}, \quad c \stackrel{\text { def }}{=} \frac{n_{x}^{2} / L_{x}^{2}}{n_{x}^{2} / L_{x}^{2}+n_{z}^{2} / L_{z}^{2}} .
$$


The streamwise- and spanwise-invariant modes therefore take the forms

$$
\begin{array}{ll}
\boldsymbol{\phi}_{0, n_{z}}^{(n)[1]}(y)=\left(\begin{array}{c}
\boldsymbol{\phi}_{1,0, n_{z}}^{(n)}(y) \\
0 \\
0
\end{array}\right), \quad \boldsymbol{\phi}_{0, n_{z}}^{(n)[2]}(y)=\left(\begin{array}{c}
0 \\
\boldsymbol{\phi}_{2,0, n_{z}}^{(n)}(y) \\
\boldsymbol{\phi}_{3,0, n_{z}}^{(n)}(y)
\end{array}\right), \\
\boldsymbol{\phi}_{n_{x}, 0}^{(n)[1]}(y)=\left(\begin{array}{c}
0 \\
0 \\
\boldsymbol{\phi}_{3, n_{x}, 0}^{(n)}(y)
\end{array}\right), & \boldsymbol{\phi}_{n_{x}, 0}^{(n)[2]}(y)=\left(\begin{array}{c}
\boldsymbol{\phi}_{1, n_{x}, 0}^{(n)}(y) \\
\boldsymbol{\phi}_{2, n_{x}, 0}^{(n)}(y) \\
0
\end{array}\right),
\end{array}
$$

and for modes with neither streamwise nor spanwise variation we have $\boldsymbol{\phi}_{0,0}^{(n)[1]}(y)=0$, $\boldsymbol{\phi}_{0,0}^{(n)[2]}(y)=\boldsymbol{\phi}_{0,0}^{(n)}(y)$. We also note that the functions are pairwise-orthogonal and divergence-free:

$$
\begin{aligned}
\left(\boldsymbol{\Phi}_{n_{x}, n_{z}}^{(n)[m]}(\boldsymbol{x}), \boldsymbol{\Phi}_{n_{x}, n_{z}}^{\left(n^{\prime}\right)\left[m^{\prime}\right]}(\boldsymbol{x})\right) & =e_{n_{x}, n_{z}}^{(n)[m]} \delta_{n n^{\prime}} \delta_{m m^{\prime}}, \\
\nabla \cdot \boldsymbol{\Phi}_{n_{x}, n_{z}}^{(n)[m]}(\boldsymbol{x}) & =0 \text { for } m=1,2 .
\end{aligned}
$$

We do not normalize the uncoupled modes, hence the (non-unity) coefficients $e_{n_{x}, n_{z}}^{(n)[m]}$; however, we have $e_{n_{x}, n_{z}}^{(n)[1]}+e_{n_{x}, n_{z}}^{(n)[2]}=1$.

For $n_{x}=0$ we recover the decomposition of Berkooz et al. (1991), but in general neither term in the decomposition represents a purely streamwise or cross-stream component. For $n_{x}, n_{z} \neq 0, \boldsymbol{\phi}_{n_{x}, n_{z}}^{[1]}(y)$ represents a structure that lies parallel with the walls at $y= \pm 1$, while $\boldsymbol{\phi}_{n_{x}, n_{z}}^{[2]}(y)$ is fully three-dimensional, cf. (4.7)-(4.8).

A recent paper by Juttijudata, Lumley \& Rempfer (2005) derives uncoupled ('plus and minus') POD modes from DNS data directly, using the transformation of Squire (1933) in Fourier space (with respect to $n_{x}, n_{z}$ ), rather than splitting each coupled POD mode after derivation, as we do. They show that large $(O(3000)$ complex ODE) Galerkin projections into subspaces of the POD plus and minus modes capture the relative energies of streamwise and spanwise turbulent fluctuations better than ones based on coupled modes. Since the orientation of the Squire coordinates changes with wavenumber vector, it is hard to compare this decomposition directly with that of Waleffe used here (cf. Juttijudata et al. 2005), but their work further supports our general aim to find less constrained low-dimensional models.

\subsection{Structure of the projected ODES}

Uncoupling the $(1,0,1),(1,0,2),(1,0,3),(1,1, \pm 1)$, and $(1,1, \pm 2)$ POD modes as described above and projecting equation (1.1) onto these modes along with the singlecomponent $(1,0,0)$ and $(1,1,0)$ POD modes, we obtain ODEs for the following set of modal amplitudes:

$$
\boldsymbol{a}^{U C}=\left(a_{0,0}^{(1)}, a_{0,1}^{(1)[1,2]}, a_{0,2}^{(1)[1,2]}, a_{1,0}^{(1)}, a_{1,1}^{(1)[1,2]}, a_{1,-1}^{(1)[1,2]}, a_{0,3}^{(1)[1,2]}, a_{1,2}^{(1)[1,2]}, a_{1,-2}^{(1)[1,2]}\right) .
$$

These equations, which we refer to as the 9-uncoupled-mode model, are too lengthy to display here, but have similar structure to the equations obtained in $\S 3$, with important differences in the linear terms to be subsequently described. $\dagger$ We note that $a_{0,0}^{(1)}$ is real, all other modal amplitudes are complex, and that all modes other than $a_{0,0}^{(1)}$ and $a_{1,0}^{(1)}$ appear in pairs. Counting each complex mode as two real dimensions, the 9-uncoupled-mode model is therefore 31-dimensional.

$\dagger$ Full listings are available as a supplement to the online version of the paper or from the first author. 

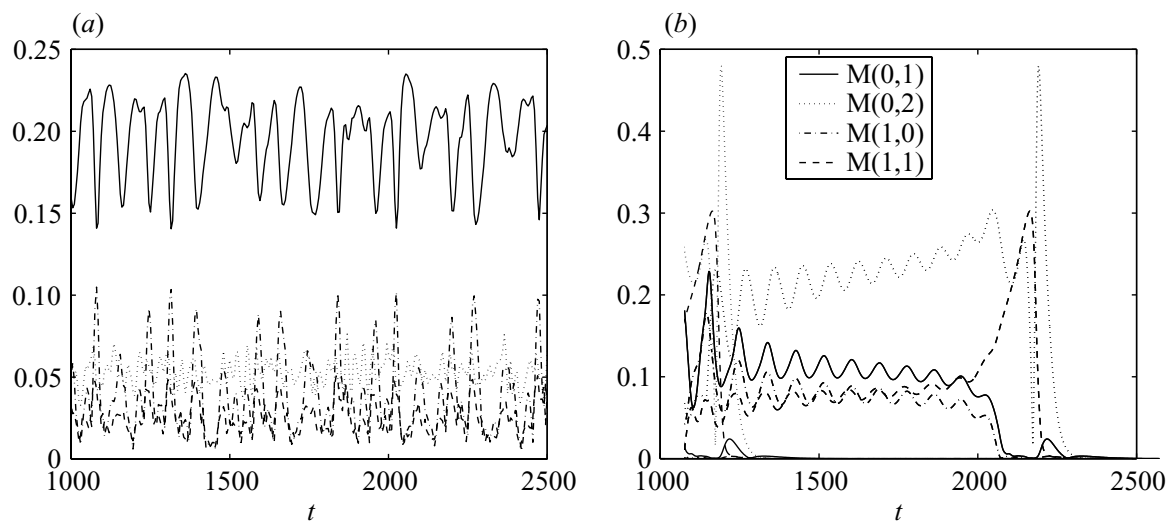

FIGURE 20. RMS modal velocities for $(a)$ the DNS and $(b)$ computed from the 6-uncoupledmode model from (4.14). Note the differing scales on the ordinates of these two plots.

\subsection{Dynamical behaviour of the models involving uncoupled modes}

\subsubsection{The 6-uncoupled-mode model}

By restricting the amplitudes $a_{0,3}^{(1)[1,2]}, a_{1,2}^{(1)[1,2]}$, and $a_{1,-2}^{(1)[1,2]}$ to be zero, we obtain a 6-uncoupled-mode model. As noted at the start of this section, for any initial condition, the solution of an uncoupled expansion with zero streamwise variation must eventually decay to zero (all streamwise-invariant flows necessarily relax to the laminar state). It was hypothesized in Berkooz et al. (1991) that, during this transient, the system would display 'ghosts' of the behaviour demonstrated in the coupled expansion. In the 6 -uncoupled-mode model, we include the $(1,1,0)$ and $(1,1, \pm 1)$ streamwise modes, which do provide a (genuine) source of energy, so one might reasonably expect sustained dynamic behaviour. In figure 20 we plot the RMS modal velocities for this uncoupled model, computed via

$$
\mathrm{M}\left(n_{x}, n_{z}\right)=\sqrt{\frac{\sum_{n} e_{n_{x}, n_{z}}^{(n)[1]}\left|a_{n_{x}, n_{z}}^{(n)[1]}\right|^{2}+e_{n_{x}, n_{z}}^{(n)[2]}\left|a_{n_{x}, n_{z}}^{(n)[2]}\right|^{2}}{L_{x} L_{z}}},
$$

and observe that this is not the case. During the transient, however, the dynamics resemble the behaviour of $\S 3.4$ with $\alpha=0.8$. In particular, the modal amplitudes are rapidly attracted to (some rotation of) the real subspace, and the transient oscillations have an approximate period of 87 non-dimensional time units.

\subsubsection{The 9-uncoupled-mode model}

We now explore the extent to which models based on uncoupled modes remedy the problem of instability of the laminar state, exhibited by the 6-coupled-mode model. Without accounting for energy losses to neglected modes, the laminar state of the 9 -uncoupled-mode model does not remain stable for all values of $R e$. Indeed, the evolution equations for $a_{1,2}^{(1)[2]}$ and $a_{1,-2}^{(1)[2]}$ reveal that the laminar solution loses stability at

$$
R e=-\frac{A_{12}^{\prime \prime \prime}}{A_{12}^{\mathrm{iv}}}=577.6,
$$

where $A_{12}^{\prime \prime \prime}$ and $A_{12}^{\mathrm{iv}}$ are the analogues of the $A_{n_{x}, n_{z}}^{\prime}, A_{n_{x}, n_{z}}^{\prime \prime}$ terms of 3.8 , cf. 4.18 below. We could argue that this is of little relevance, since the POD modes are derived from 

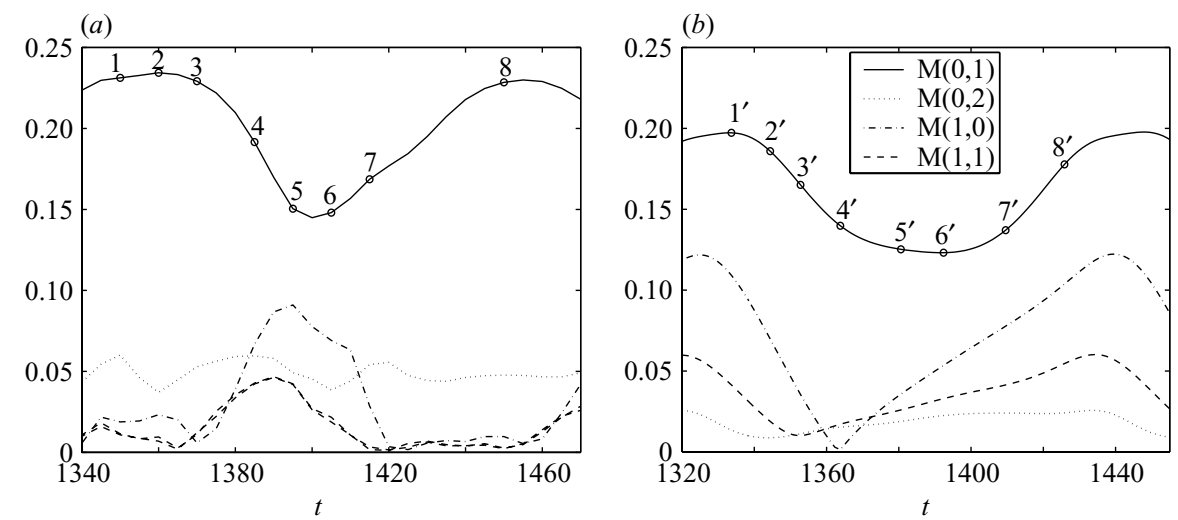

FIGURE 21. RMS modal velocities: $(a)$ from a representative cycle of the DNS (repeated from figure 10) and (b) computed from one period of the 9-uncoupled-mode model with $\alpha=0.22$.

data at $R e=400$, but more significantly, it may be remedied by adding eddy viscosity terms. Slightly generalizing the procedure of $\S 3.3$, we therefore add a term of the form

$$
-\alpha v\left(n_{x}^{2}+n_{z}^{2}\right) a_{n_{x}, n_{z}}^{(n)[m]}
$$

to each ODE, and find that the laminar state remains stable for all Re provided that we choose $\alpha$ to satisfy

$$
\alpha>\frac{A_{12}^{\mathrm{iv}}}{5 v e_{12}^{[2]}}=0.2179
$$

(we retain $\nu=0.0333$ as in the 6-coupled-mode model). We set $\alpha=0.22$ and compute bifurcation diagrams for steady and periodic states with respect to $R e$, with a view to comparisons with branches of steady solutions of the Navier-Stokes equations, noting that this value is not special: similar results apply for other nearby values of $\alpha$.

\subsubsection{Dominant attractor at $R e=400$}

The laminar state is now stable, and solutions started sufficiently close to it approach it as $t \rightarrow \infty$. However, at $R e=400$ almost all initial conditions of significant amplitude approach a stable periodic orbit lying within the subspace in which $a_{1,-1}^{(1)[1,2]}=-a_{1,1}^{(1)[1,2]}$ and $a_{1,-2}^{(1)[1,2]}=a_{1,2}^{(1)[1,2]}$. This is analogous to the limit cycle of the 6-coupled-mode model with losses (figures 14-17), but has a period of 228.5 non-dimensional time units: over twice the recurrence period of streak breakdown from DNS simulations. However, the most energetic modes, including those most prominent in the streak breakdown process, oscillate with half this period, giving reasonable agreement, as shown in figures 21-23. To explore the source of this attractor, we use AUTO (Doedel et al. 1997) to follow its locus over a range of Reynolds numbers. We find that it arises in a saddle-node bifurcation at $R e \approx 379.8$ and loses stability in another saddle-node bifurcation at $R e \approx 852.8$, forming an isola (Smith 2003). The remainder of the branch, and additional branches bifurcating from it, are all unstable. This is consistent with the observation that the MFU turbulent state appears as the Reynolds numbers passes from 300 to 400 , cf. HKW.

RMS modal velocities for this model, calculated via (4.14), are compared with the same quantities for DNS in figure 21. Whilst magnitudes agree fairly well, phase relationships among the model's modal velocities are incorrect; in particular, 


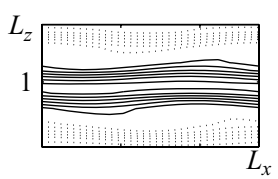

(a)
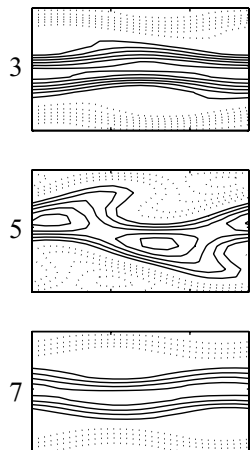
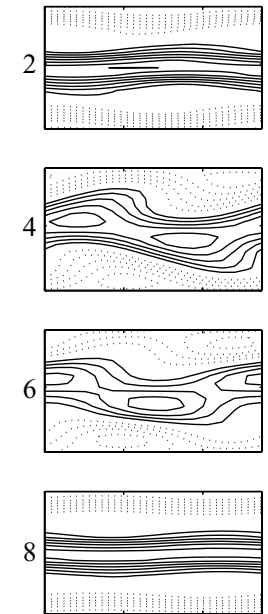

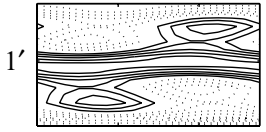

(b)
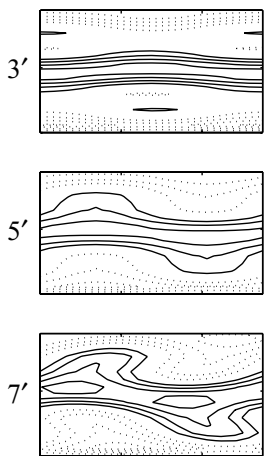
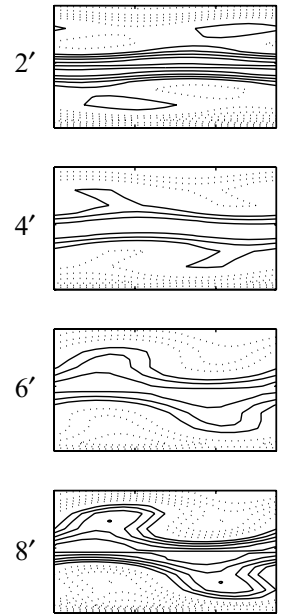

FIGURE 22. The streak breakdown process as viewed in the $(x, z)$-plane lying between the two plates in PCF: (a) DNS projected onto the 9 modes present in the model and $(b)$ computed from one period of the 9-uncoupled-mode model with $\alpha=0.22$.

(a)

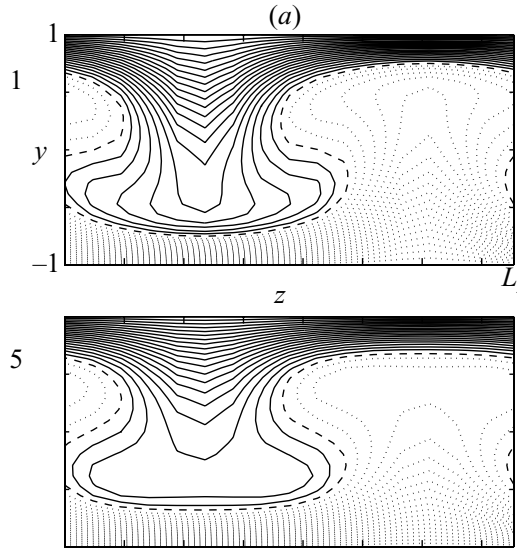

(b)
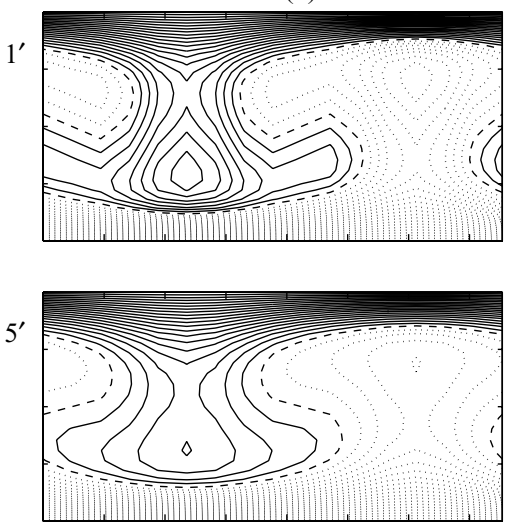

FIGURE 23. Streamwise velocities in the $(y, z)$-plane calculated from the streamwise-invariant modes and the laminar state: $(a)$ DNS projected onto the 9 modes present in the model and (b) computed from one period of the 9-uncoupled-mode model with $\alpha=0.22$ (right).

$\mathrm{M}(0,1)$ and $\mathrm{M}(1,0)$ are now in phase and the model streak-breakdown process is consequently phase-shifted, as shown in figure 22. The velocity contours resulting from the streamwise-invariant modes in the model, shown in figure 23, are however much improved; here there is no need to subtract the laminar state to demonstrate qualitative agreement between model and DNS results.

In figure 24 we plot turbulence statistics for the 9-uncoupled-mode model. In comparison to the analogous quantities for the 6-coupled-mode model of figure 19, the RMS $u^{\prime}$ velocity is slightly improved, the RMS $v^{\prime}$ velocity is now larger than the DNS data rather than smaller, and while the RMS $w^{\prime}$ velocity is significantly greater (and worse) in magnitude, it now has the correct form, with a central trough rather than a peak. The Reynolds stress, bottom right, is also improved, showing very good agreement between DNS and model in the near-wall region, for average Reynolds 

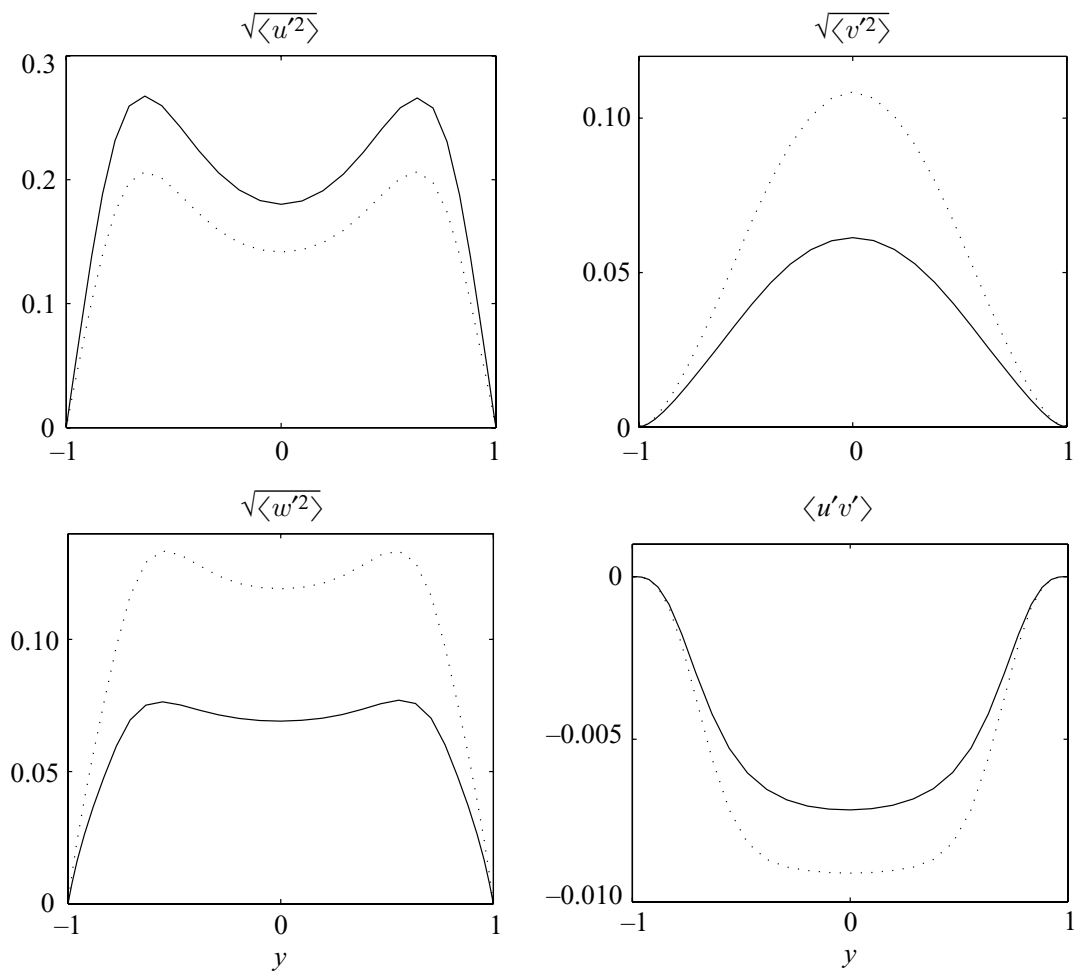

FigURE 24. Performance of the 9-uncoupled-mode model with $\alpha=0.22$ measured by turbulent statistics with DNS (solid) and model (dotted) shown.

stress $\left(\left\langle u^{\prime} v^{\prime}\right\rangle\right.$ ). (As for the 6-coupled-mode model, agreement between instantaneous Reynolds stress from the DNS and model is best when streaks are prominent; it is poorer during the streak breakdown process, because the model does not resolve the small structures and mixing processes.)

In addition to this periodic orbit, there are many other periodic and stationary states; some of the latter are shown in figure 25. With the exception of the stable laminar state represented by the solid line with amplitude $\mathscr{A}=0$, these fixed points are generated in saddle-node bifurcations, predominantly in the range $R e=200-375$, and they are all unstable with the exception of a single (modulo symmetry) stable state born in a saddle-node bifurcation at $R e \approx 220$, that becomes unstable in a supercritical Hopf bifurcation at $R e \approx 393$ ). These branches are qualitatively similar to those found by Schmiegel (1999) for the full Navier-Stokes equations, cf. Nagata (1990) and Clever \& Busse (1992).

\subsection{Non-normality effects in the uncoupled models}

As noted in $\S 1$, the linear operator in (1.1) is non-normal; however, the constraints among velocity components implicit in restriction to the first family of quantum numbers $n=1$ in the coupled mode models of $\S 3$ render the linear parts of (3.7) diagonal (and hence very normal). Our uncoupled expansion restores non-normality, typical pairs of model coefficients now being linked by upper triangular blocks in the 

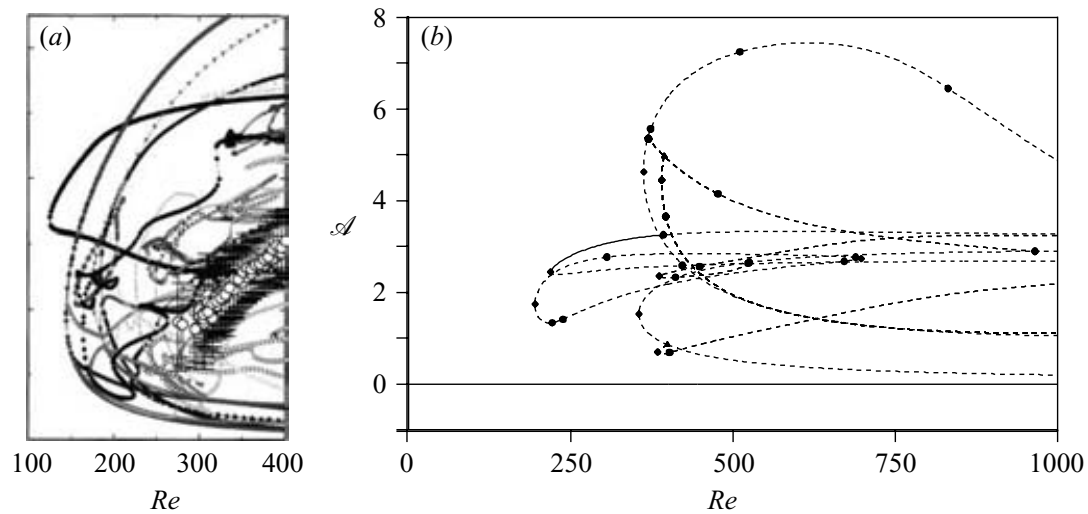

Figure 25. (a) Branches of fixed points in PCF with $L_{x}=4 \pi$ and $L_{z}=2 \pi$, from Schmiegel (1999) adapted from Faisst et al. (2000), cf. Schmiegel (1999). (b) Branches of fixed points in the full phase space of the 9 -uncoupled-mode model with $\alpha=0.22$; the ordinate $\mathscr{A}$ denotes the $L^{2}$ norm and the stable laminar solution is represented by the solid line at $\mathscr{A}=0$. Solid (resp., dashed) lines indicate stable (resp., unstable) solutions, and the dots indicate bifurcation points.

linear part $A^{U C}$ of the projected equations:

$$
\left.\begin{array}{l}
\dot{a}_{n_{x}, n_{z}}^{(1)[1]}=\left(A_{n_{x} n_{y}}^{\prime} / R e\right) a_{n_{x}, n_{z}}^{(1)[1]}+A_{n_{x} n_{y}}^{\prime \prime} a_{n_{x}, n_{z}}^{(1)[2]}+\cdots, \\
\dot{a}_{n_{x}, n_{z}}^{(1)[2]}=\left(A_{n_{x} n_{y}}^{\prime \prime \prime} / R e+A_{n_{x} n_{y}}^{\mathrm{iv}}\right) a_{n_{x}, n_{z}}^{(1)[2]}+\cdots
\end{array}\right\}
$$

(cf. Smith 2003). Trefethen (1992)'s pseudospectral theory shows that stable but highly non-normal operators with eigenvalues close to the imaginary axis are likely to have $\epsilon$-pseudospectra contours protruding into the right-hand half-plane, and Trefethen et al. (1993) show that this holds for plane Couette flow at high Reynolds numbers and for Poiseuille flow at Reynolds numbers slightly less than the critical value 5772 . Hence for the linearized system certain modes can grow very large before eventually decaying, suggesting that small perturbations from the laminar state might trigger a (nonlinear) transition to sustained turbulence.

We found that $\epsilon$-pseudospectra contours for the linear part of the 9-uncoupledmode model do not extend far into the right-hand half-plane, but still investigated the dynamical effects of non-normality via numerical simulations by plotting the energy of the 9-uncoupled-mode model,

$$
\mathscr{E}(t)=\sum e_{n_{x}, n_{z}}^{(n)[m]}\left|a_{n_{x}, n_{z}}^{(n)[m]}\right|^{2},
$$

where the sum is over the modes retained, during transient samples for $R e=400$ and $\alpha=0.22$; see figure 26. In panel $(a)$ we follow the prescription in Baggett $\&$ Trefethen (1997, § III), setting

$$
\boldsymbol{a}^{U C}(0)=C\left(\boldsymbol{a}_{\text {fitg }}^{U C}+0.1 \boldsymbol{a}_{\mathrm{rand}}^{U C}\right),
$$

where $\boldsymbol{a}_{\text {fitg }}^{U C}$ is a unit vector aligned with the fastest initial transient growth associated with $A^{U C}$, found through maximization of $(\mathrm{d} / \mathrm{d} t)\left\|\mathrm{e}^{t A^{U C}} \boldsymbol{a}_{\text {fitg }}^{U C}\right\|$ at $t=0$ subject to the constraint $\left\|\boldsymbol{a}_{\text {fitg }}^{U C}\right\|=1$. Obtaining this via the eigenvalue decomposition of $A^{U C}+\left(A^{U C}\right)^{T}$ indicates that $\boldsymbol{a}_{\text {fitg }}^{U C}$ lies in the $a_{1, \pm 2}^{(1)[1,2]}$ subspace. We also found a numerical approximation to the direction in this subspace which leads to maximum linear transient growth, 

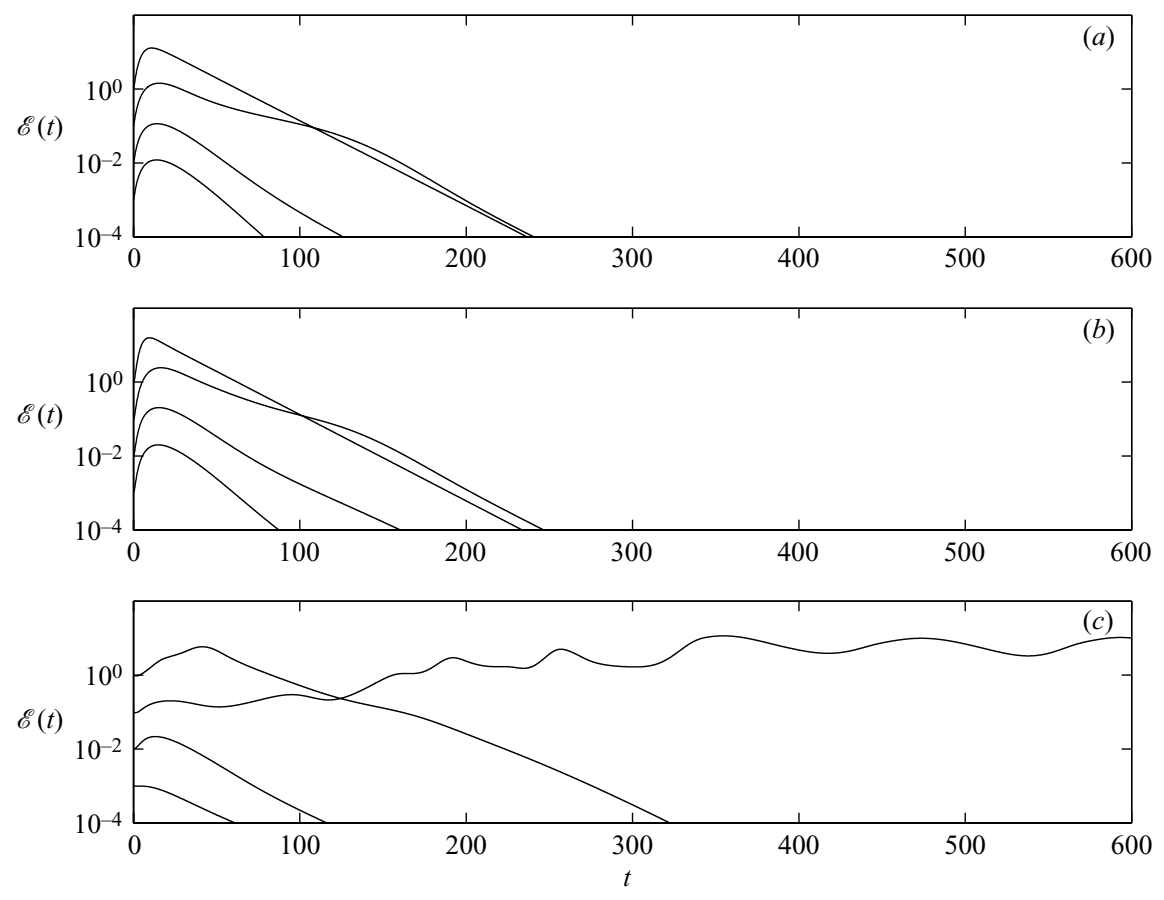

FIGURE 26. Transient behaviour of the 9-uncoupled-mode model $(R e=400, \alpha=0.22)$ in the real subspace. In each of the three panels we plot the model's energy $\mathscr{E}(t)$, as given in (4.19), with initial values of $10^{-3}, 10^{-2}, 10^{-1}$ and 1 . In $(a)$ initial conditions are chosen to be parallel to the direction specified by (4.20), in $(b)$ they are chosen to be parallel to the direction specified by (4.21), while in $(c)$ they are generated from purely random data.

denoted $\boldsymbol{a}_{\mathrm{mltg}}^{U C}$, and in figure 26(b) we set initial conditions

$$
\boldsymbol{a}^{U C}(0)=C\left(\boldsymbol{a}_{\mathrm{mltg}}^{U C}+0.1 \boldsymbol{a}_{\mathrm{rand}}^{U C}\right) \text {. }
$$

In both cases a random unit vector $\boldsymbol{a}_{\text {rand }}^{U C}$ is added and the sum scaled by a constant $C$. In figure 26(c) we choose the initial condition vector solely at random. The results in figure 26 are representative of those found over many runs. Interestingly, the only trajectory which shows sustained turbulence is that corresponding to random initial data with $\mathscr{E}(0)$ set to $10^{-1}$.

In figure 27 we show DNS transients for the same initial conditions as those of figure 26. The agreement is reasonable, with sustained turbulence occurring for random data with initial energies $\mathscr{E}(0)=10^{-1}$ and 1.0 , although decay to the laminar state is somewhat slower for the DNS.

The model also predicts, and DNS confirms, that the linear interaction between the amplitudes $a_{0,1}^{(1)[1]}$ and $a_{0,1}^{(1)[2]}$ can give transient growth. This is associated with the interaction between streamwise vortices and streaks, and involves the vortex tilting mechanism described in Butler \& Farrell (1992).

We thus conclude that, while non-normality may result in a modest transient amplification of suitably aligned initial conditions, this alone is generally insufficient to guarantee transition to the 'turbulent' state. Indeed, the boundaries of the domains of attraction of both the laminar state and the periodic orbit are likely to be complicated sets within the full phase space, and amplification in any one direction need not necessarily cause transition. Schmiegel \& Eckhardt (1997) and Eckhardt \& 

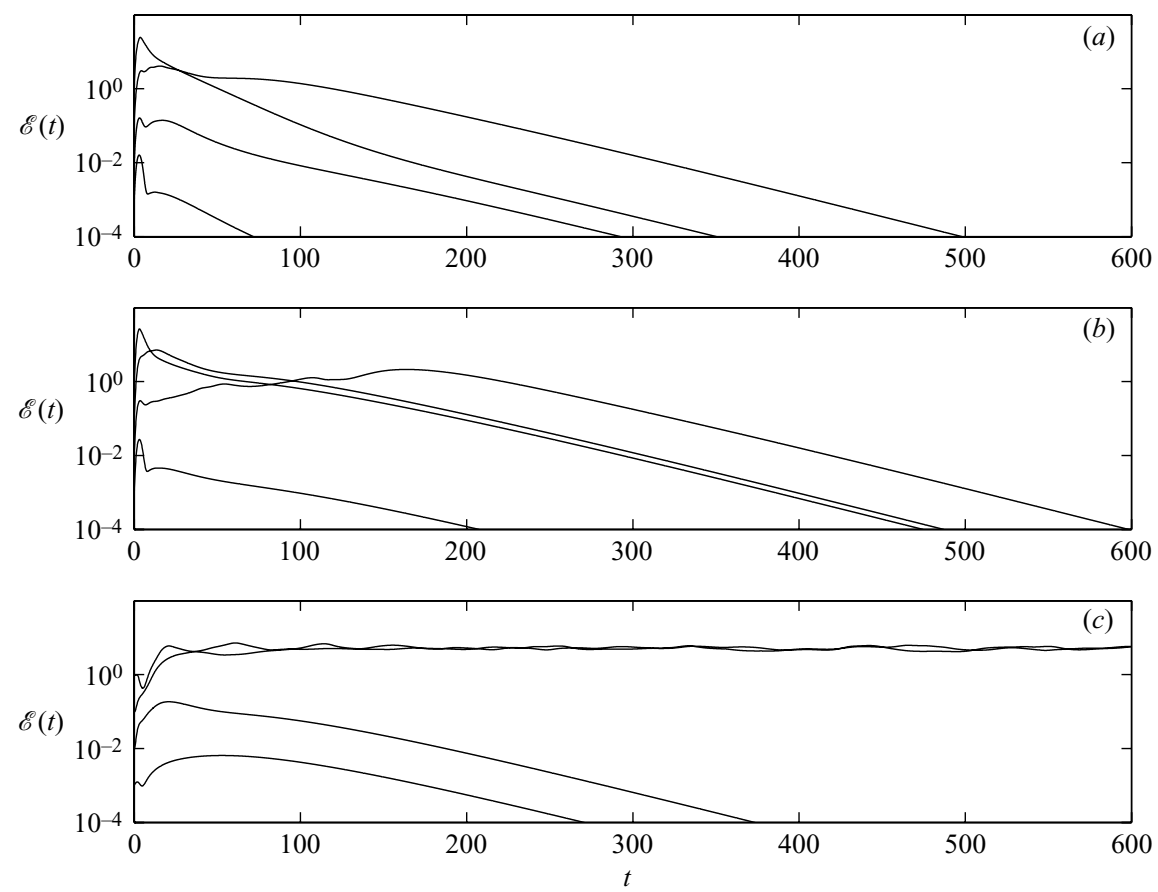

FIGURE 27. Transient behaviour of the DNS $(R e=400)$. Once again $\mathscr{E}(t)$ is calculated according to (4.19). and is plotted for initial conditions, in each panel, identical to those in the respective panels of figure 26.

Mersmann (1999) have shown that the full Navier-Stokes equations (1.1) apparently exhibit fractal domain boundaries. With this in mind, it is unclear what linear analysis of the laminar state, normal or not, can reveal about transition.

Simulations with varying initial data uncover another interesting feature. As figure 26 indicates, for $\alpha=0.22$ most trajectories in the 9-uncoupled-mode model either decay 'smoothly' to zero or converge on the periodic orbit of figure 21, but some initial conditions lead to long-lived transients, presumably while solutions negotiate the fractal boundaries, as illustrated in figure 28. Here panel $(a)$ shows $\mathscr{E}(t)$ on a normal Cartesian scale (with $\mathscr{E}(0)=10^{-1}$ ) and in panel $(b)$ we reconstruct the RMS modal velocities of (4.14). For several thousand time units the behaviour is strikingly similar to that of RMS modal velocities of the DNS (cf. figure 2), before collapsing the origin rather abruptly. This is reminiscent of numerical simulations of $\mathrm{PCF}$ in cases in which the spanwise dimension has been reduced to a value too small to sustain turbulence, e.g. as in HKW figure 20. We observed similar behaviour in our DNS simulations of the standard MFU domain for various initial conditions before arriving at the sustained behaviour of figure 2 .

\section{Conclusion}

We open this concluding section with brief surveys of other low-dimensional models of shear-driven instability and turbulence, and then summarize our principal findings. 

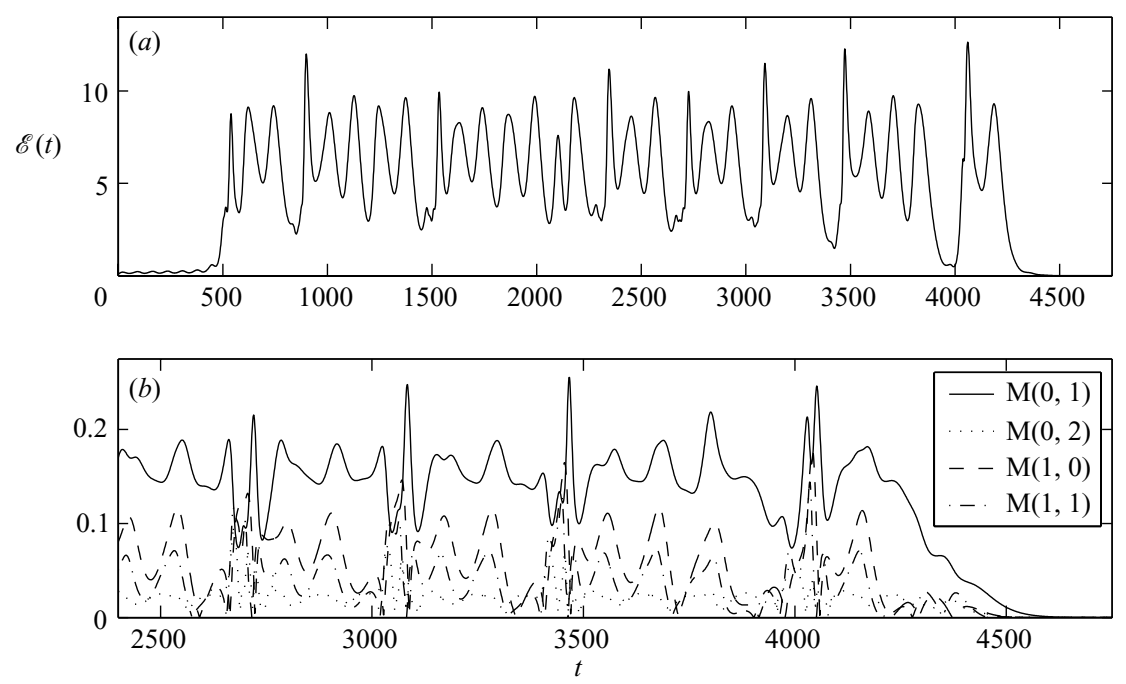

FIGURE 28. Transient behaviour of the 9-uncoupled-mode model in the real subspace with $R e=400$ and $\alpha=0.22$. Initial energy is set to $\mathscr{E}(0)=10^{-1}$. In $(a) \mathscr{E}(t)$ is plotted; in $(b)$ we reconstruct the RMS modal velocities according to (4.14). This is to be compared with the behaviour in figure 21 .

\subsection{Waleffe's models}

Waleffe proposed two models of turbulent PCF in the papers Waleffe $(1995 a, b, 1997)$ that followed HKW. Both are 4-dimensional real quadratic ODEs of the form:

$$
\frac{\mathrm{d}}{\mathrm{d} t}\left(\begin{array}{c}
u \\
v \\
w \\
m
\end{array}\right)=\frac{1}{R e}\left(\begin{array}{c}
\lambda_{u} u \\
\lambda_{v} v \\
\lambda_{w} w \\
\lambda_{m} m-\sigma_{m}
\end{array}\right)+\left(\begin{array}{cccc}
0 & 0 & -\sigma_{w} w & \sigma_{u} v \\
0 & 0 & \sigma_{v} w & 0 \\
\sigma_{w} w & -\sigma_{v} w & 0 & -\sigma_{m} w \\
-\sigma_{u} v & 0 & \sigma_{m} w & 0
\end{array}\right)\left(\begin{array}{c}
u \\
v \\
w \\
m
\end{array}\right)
$$

where the variables $(u, v, w, m)$ respectively represent the amplitudes of spanwise modulation of the streamwise velocity, of the spanwise rolls, of an inflectional streak instability, and of the mean shear. Explicit expressions for the spatial fields of these modes are not given.

Waleffe $(1995 b)$ sets the parameters to $\left[\sigma_{m}, \sigma_{u}, \sigma_{v}, \sigma_{w}\right]=[0,1,1,0.5]$ and $\left[\lambda_{m}, \lambda_{u}, \lambda_{v}, \lambda_{w}\right]=[-10,-10,-10,-15]:$ this guarantees that the laminar state $(u, v, w, m)=(0,0,0,1)$ is stable for all values of $R e$, and the bifurcation behaviour of (5.1) is then as follows. A saddle-node bifurcation of fixed points occurs at $R e=98.6325$, creating stable and unstable steady solutions. The lower branch is unstable for all moderate values of $R e$; the initially stable upper branch becomes unstable in a supercritical Hopf bifurcation at $R e=100.0232$ and the resulting periodic orbit remains stable until it disappears in a homoclinic bifurcation at $R e=101.0311$. The laminar state is the only stable attractor in the range $101.0311<R e<356$, after which another stable periodic orbit appears in a second homoclinic bifurcation, remaining stable until it vanishes in a third homoclinic bifurcation at $R e \approx 435$ : a time series for this periodic orbit at $R e=400$ is shown in figure 29. Given the definitions of $u$ and $v$ above, one might infer that the RMS modal velocities associated with (5.1) can be expressed (in terms of 2.1) as $v \sim \mathrm{M}(0,1)$ and $u \sim \mathrm{M}(1,0)$, in which case these would be in phase rather than antiphase, as in the MFU of HKW (cf. figure 2). 


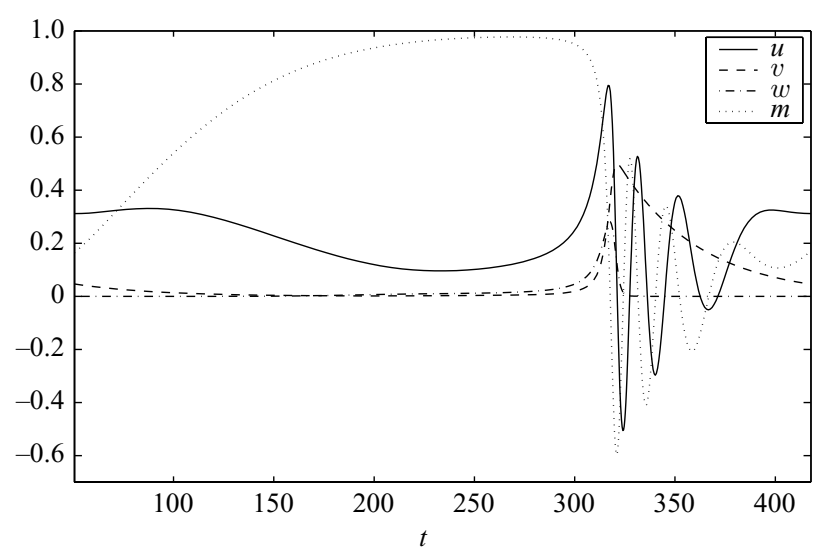

FIGURE 29. Behaviour of the periodic orbit of (5.1).

A similar model is considered in Waleffe (1997), which attempts to put (5.1) on firmer footing. Waleffe begins by considering sinusoidal shear flow - a channel subject to sinusoidal forcing with free-slip at the bounding walls - which is related, but not identical, to PCF. This (artificial) flow has the advantage that elementary trigonometric basis functions exist, yielding ODEs with analytically determined coefficients. Waleffe projects the Navier-Stokes equations onto eight (real) modes, and then imposes constraints among modal amplitudes to reduce first to a 5- and ultimately a 4-dimensional model. Although the rationale for these constraints seems unclear, the final result is a model 'derived' from Navier-Stokes, with parameters $\left[\sigma_{m}, \sigma_{u}, \sigma_{v}, \sigma_{w}\right]=$ $[0.31,1.29 .0 .22,0.68]$ and $\left[\lambda_{m}, \lambda_{u}, \lambda_{v}, \lambda_{w}\right]=[2.47,5.20,7.67,7.13]$. The most significant difference between this model and that in Waleffe $(1995 a, b)$ is the inclusion of a non-zero $\sigma_{m}$ term, representing interaction between the mean shear and the streak instability. Waleffe (1997, p. 894), then notes that '... an unstable limit cycle is generated... for the parameter values of interest'. This observation is supported by Dauchot \& Vioujard's study of the system (Dauchot \& Vioujard 2000), in which figure 2 shows a saddle-node bifurcation which gives two unstable fixed points; one of these subsequently gains stability in a subcritical Hopf bifurcation in which an unstable periodic orbit branch appears. Dauchot \& Vioujard discover regions of parameter space for which either no Hopf bifurcation occurs or a subcritical Hopf bifurcation occurs, yielding unstable periodic orbits. Nowhere is it claimed that the Hopf bifurcation is supercritical or stable periodic orbits exist, and parameter ranges in which the homoclinic bifurcation of Waleffe $(1995 a, b)$ might occur are not identified. Waleffe (1997) ends by quoting his previous stable periodic orbit, which does not exist for the parameters derived in Waleffe (1997), and Waleffe (1997, figure 13) shows 'some possible dynamics' for the four-dimensional model. Thus, while 5.1 contains ingredients that can result in a regeneration cycle, parameter values for which this occurs do not seem derivable from fluid physics.

\subsection{Models of the Universität Marburg group}

Several low-dimensional models inspired by PCF have been considered by $\mathrm{B}$. Eckhardt's group at the Universität Marburg, as detailed in Eckhardt \& Mersmann (1999), Schmiegel \& Eckhardt (1997) and Schmiegel (1999). As in Waleffe (1997), these models were also constructed for sinusoidal shear flow. The most interesting among them have 9 and 19 degrees of freedom, and both exhibit transitional behaviour 
similar to PCF. In Eckhardt \& Mersmann (1999) the evolution of perturbations for the 19 -mode model is displayed in a plot similar to those of figure 26 above (although the critical perturbation amplitudes differ greatly). Similar information for the 9-mode model, which is essentially the 19-mode model restricted to an invariant subspace, is presented in three-dimensional form in Schmiegel (1999). In both instances, the fractal nature of the lifetime of perturbations is investigated; something which we did not address in this paper.

Various stationary states appear as $R e$ is increased in the 19-mode model, the first in a saddle-node bifurcation at $R e \approx 190$. This is similar to full Navier-Stokes results for PCF in Nagata (1990) and Clever \& Busse (1992) (Re $\approx 125)$, and also to the present uncoupled 16-mode model of $\S 4.3$ ( $R e \approx 195$ : figure 25$)$. In Schmiegel (1999) it is concluded that these stationary states have no dynamic significance, and instead it is collections of unstable periodic orbits which lead to the transition to turbulence by means of a 'chaotic repeller'. This contrasts with evidence that PCF turbulence arises through stationary states and heteroclinic orbits among them, also presented in Schmiegel (1999). We recall that Moehlis et al. (2002) also found, for the moderate aspect ratio domain, various complicated dynamics deriving from heteroclinic connections among stationary states. Schmiegel reasons that these lowdimensional models and the full PCF problem differ due to boundary conditions (free-slip for the sinusoidal shear flow of the models, no-slip for PCF). Moehlis et al. $(2004,2005)$ also find that collections of unstable periodic orbits are associated with a chaotic repellor and turbulence for a different 9-mode model for sinusoidal shear flow, which generalizes the 8-mode model of Waleffe (1997).

\subsection{Summary of the present results}

We believe that our attempts to model plane Couette flow in the minimal flow unit have been a qualified success. We have examined two models in depth: one constructed from six and the other from nine POD modes. At Reynolds number 400, the 6-coupled-mode model has simple travelling waves with amplitudes that well-approximate the corresponding average DNS quantities. Since the POD is a dynamics-blind, statistical technique one might conclude that this is the best that can be done. However, on adding damping terms to represent energy losses to neglected modes, the travelling waves are replaced by periodic orbits confined to (a rotation of) the real subspace that shows good qualitative agreement with DNS statistics and quantitative agreement with DNS time scales. Additionally, velocity field reconstructions analogous to those of HKW also agree with DNS fields, at least for perturbations riding on the laminar solution.

We also derived and analysed uncoupled POD models, as suggested by Berkooz et al. (1991) and independently by Waleffe (1995 b), and simultaneously approached from a different viewpoint by Juttijudata et al. (2005). We found that Berkooz et al. (1991)'s conjecture that models involving uncoupled modes would retain 'ghosts' of the behaviour of models based on coupled modes is not strictly true: the 6-uncoupledmode model gives unsustained behaviour, although we can relate it to the behaviour of the damped 6-coupled-mode model. In contrast, the 9-mode truncation, which did not lead to an interesting dynamic model with coupled modes, does yield a reasonable model when the modes are suitably uncoupled. Moreover, we can remedy the problem of instability of the laminar state for models involving coupled modes, while retaining the cyclic regeneration behaviour. We also examined the influence of non-normality of the linear terms in the 9-uncoupled-mode model, finding that while it does weakly 
determine the initial response to perturbations, it is a poor indicator of whether the perturbations lead to 'transition' to the non-trivial state, or collapse to laminar flow.

This work, along with Berkooz et al. (1991), shows that uncoupled modes can remove constraints imposed by very low-dimensional projections that can lead to incorrect behavior - failure of streamwise-invariant perturbations to decay, and (for PCF) instability of the laminar state and lack of non-normality - but it does not imply that all low-dimensional models should employ them. As argued in Holmes et al. (1996), even coupled models lacking streamwise modes such as that of Aubry et al. (1988) can illuminate important physics, provided that their limitations are understood. Also, as shown in $\$ 3.1$ and Gibson (2002), high-dimensional POD truncations that include streamwise modes do not suffer from the relative amplitude constraints due to the larger numbers of modes available.

In both the 6-coupled-mode and 9-uncoupled-mode models, the attractor most relevant to the regeneration cycle is a standing-wave periodic orbit. The projection of this onto the three dominant modes $((1,0,0),(1,0,1)$ and $(1,0,2))$ reveals that it involves a cyclic transfer of energy among the mean flow $\sim(1,0,0)$ (figure 6 ) and streamwise-invariant vortical structures with differing cross-stream scales $(1,0,1),(1,0,2)$ (figure 7$)$. However, although these three modes capture $93.5 \%$ of the energy on average, at least three further modes (albeit containing less than $1.3 \%$ ) are required to sustain the turbulent dynamics; notably, these include the streamwisevarying $(1,1,0)$ and $(1,1, \pm 1)$ modes. As pointed out by Zhou \& Sirovich (1992), Sanghi \& Aubry (1993) and Webber et al. (1997), modes with streamwise variation have significant influence on the dynamics. The 6-coupled-mode model reproduces the relative phases of $n_{x}=0$ roll and $n_{x} \neq 0$ propagating modes acceptably, yielding velocity fields similar to those obtained by projecting DNS data onto the same modes (figures 15-18), and showing that it can capture the essential dynamics of the regeneration cycle. The 9-uncoupled-mode model is less successful: the phase relationships of figure 21 are incorrect, but the relative magnitudes of streamwise and cross-stream velocity components are improved (figures 22-23).

As we have noted, a motivation for developing low-dimensional models for turbulence is that novel control strategies can be more easily considered. For example, Smith et al. (2003) uses the 6-coupled mode model to suggest how streak breakdown events can be enhanced or delayed through appropriately timed impulsive perturbations. A detailed discussion of this strategy is deferred to future work.

The most striking qualitative deficiency of both models is their inability to reproduce the irregularity of the regeneration cycle, which appears in projections onto the $(1,0,1)$ and $(1,0,2)$ modes as a rapid radial oscillation precessing slowly in the azimuthal direction (cf. figures $9(b)$ and $14(b)$ ). However, in studies of the 0:1:2 spatial model we have found combinations of parameters $A_{n_{x}, n_{y}}$ at which standing waves and modulated travelling waves coalesce in 'branch-point' bifurcations, giving a potential for such behaviour (Smith et al. 2005). Hence these three dominant modes, suitably damped and excited by a model for the neglected modes, might capture the regeneration cycle both qualitatively and quantitatively.

The moral of this paper is that low-dimensional modelling is an imperfect science or, perhaps more properly, an art. While the POD necessarily yields sets of modes that contain the majority of the average turbulent kinetic energy, it is a rather poor indicator of which modes are essential to the dynamics. Despite this, through judicious selection of model truncations, use of uncoupled modes, and appropriate modelling of losses to neglected modes, one may use this technique to construct convincing 
low-dimensional models, the components (amplitudes and coefficients) of which derive directly from the Navier-Stokes equations.

This work was supported by DoE: DE-FG02-95ER25238 (T.S. and P.H.) and a National Science Foundation Mathematical Sciences Postdoctoral Research Fellowship to J.M. We thank Clancy Rowley for allowing the use and modification of his channel flow code, and the referees for their perceptive comments.

\section{REFERENCES}

Aubry, N., Holmes, P., Lumley, J. L. \& Stone, E. 1988 The dynamics of coherent structures in the wall region of the turbulent boundary layer. J. Fluid Mech. 192, 115-173.

Aubry, N., Lian, W.-Y. \& Titi, E. S. 1993 Preserving symmetries in the proper orthogonal decomposition. SIAM J. Sci. Comput. 14, 483-505.

Baggett, J. S. \& Trefethen, L. N. 1997 Low-dimensional models of subcritical transition to turbulence. Phys. Fluids 9, 1043-1053.

Barkley, D. \& Tuckerman, L. S. 1999 Stability analysis of perturbed plane Couette flow. Phys. Fluids 11, 1187-1195.

Bech, K. H., Tillmark, N., Alfredsson, P. H. \& Andersson, H. I. 1995 An investigation of turbulent plane Couette flow at low Reynolds numbers. J. Fluid Mech. 286, 291-325.

Berkooz, G., Holmes, P. \& Lumley, J. L. 1991 Intermittent dynamics in simple models of the wall layer. J. Fluid Mech. 230, 75-95.

Berkooz, G., Holmes, P. \& Lumley, J. L. 1993 The proper orthogonal decomposition in the analysis of turbulent flows. Annu. Rev. Fluid Mech. 25, 539-575.

Berkooz, G., Holmes, P., Lumley, J. L., Aubry, N. \& Stone, E. 1994 Observations regarding 'Coherence and chaos in a model of a turbulent boundary layer' by X. Zhou and L. Sirovich. Phys. Fluids 6, 1574-1578.

Berkooz, G. \& Titi, E. S. 1993 Galerkin projections and the proper orthogonal decomposition for equivariant equations. Phys. Lett. A 174, 94-102.

Bottin, S., Dauchot, O. \& Daviaud, F. 1997 Intermittency in a locally forced plane Couette flow. Phys. Rev. Lett. 79, 4377-4380.

Bottin, S., Dauchot, O., Daviaud, F. \& Manneville, P. 1998 Experimental evidence of streamwise vortices as finite amplitude solutions in transitional plane Couette flow. Phys. Fluids 10, 2597-2607.

Butler, K. M. \& Farrell, B. F. 1992 Three-dimensional optimal perturbations in viscous shear flow. Phys. Fluids A 4, 1637-1650.

Clever, R. M. \& Busse, F. H. 1992 Three-dimensional convection in a horizontal fluid layer subjected to a constant shear. J. Fluid Mech. 234, 511-527.

Coller, B. D. \& Holmes, P. 1997 Suppression of bursting. Automatica 33, 1-11.

Coller, B. D., Holmes, P. \& Lumley, J. 1994 Interaction of adjacent bursts in the wall region. Phys. Fluids 6, 954-961.

Dauchot, O. \& Daviaud, F. 1995 a Finite amplitude perturbation and spots growth mechanism in plane Couette flow. Phys. Fluids 7, 335-343.

Dauchot, O. \& Daviaud, F. $1995 b$ Streamwise vortices in plane Couette flow. Phys. Fluids 7 , 901-903.

Dauchot, O. \& Vioujard, N. 2000 Phase space analysis of a dynamical model for the subcritical transition to turbulence in plane Couette flow. Eur. Phys. J. B 14, 377-381.

Doedel, E., Champneys, A., Fairgrieve, T., Kuznetsov, Y., Sandstede, B. \& Wang, X. 1997 AUtO 97: Continuation and Bifurcation Software for Ordinary Differential Equations. Available via FTP from directory /pub/doedel/auto at ftp.cs.concordia.ca.

Drazin, P. G. \& ReID, W. H. 1981 Hydrodynamic Stability. Cambridge University Press.

Eckhardt, B. \& Mersmann, A. 1999 Transition to turbulence in a shear flow. Phys. Rev. E 60, 509-517.

FAisst, H. Schmiegel, A. \& Eckhardt, B. 2000 Dynamics of perturbations in plane Couette flow. Advances in Turbulence VIII, Proc. 8th European Turbulence Conference. (ed. C. Dopazo), pp. 71-74. CIMNE, Barcelona. 
Gibson, J. 2002 Dynamical systems models of wall-bounded, shear-flow turbulence. PhD thesis, Cornell University.

Hamilton, J., Kim, J. \& Waleffe, F. 1995 Regeneration mechanisms of near-wall turbulence structures. J. Fluid Mech. 287, 317-348 (referred to herein as HKW).

Holmes, P. 1990 Can dynamical systems approach turbulence? In Whither Turbulence? Turbulence at the Crossroads (ed. J. L. Lumley), pp. 195-249. Springer.

Holmes, P., Lumley, J. L. \& Berkooz, G. 1996 Turbulence, Coherent Structures, Dynamical Systems and Symmetry. Cambridge University Press.

Jimenez, J. \& Morn, P. 1991 The minimal flow unit in near-wall turbulence. J. Fluid Mech. 225, 213-240.

JimÉnez, J. \& Pinelli, A. 1999 The autonomous cycle of near-wall turbulence. J. Fluid Mech. 389, 335-359.

Juttijudata, V., Lumley, J. L. \& Rempfer, D. 2005 Proper orthogonal decomposition in Squire's coordinate system for dynamical models of channel turbulence. J. Fluid Mech. 534, 195-225.

KaWahara, G. \& Kida, S. 2001 Periodic motion embedded in plane Couette turbulence: regeneration cycle and burst. J. Fluid Mech. 449, 291-300.

Kim, J., Moin, P. \& Moser, R. 1987 Turbulent statistics in fully developed channel flow at low Reynolds number. J. Fluid Mech. 177, 133-166.

KLINE, S. J. 1967 The structure of turbulent boundary layers. J. Fluid Mech. 30, 741-773.

KNight, B. \& Sirovich, L. 1990 Kolmogorov inertial range for inhomogeneous turbulent flows. Phys. Rev. Lett. 65, 1356-1359.

Komminaho, J., Lundbladh, A. \& Johansson, A. V. 1996 Very large structures in plane turbulent Couette flow. J. Fluid Mech. 320, 259-285.

Lumley, J. L. 1971 Stochastic Tools in Turbulence. Academic.

Moehlis, J., Faisst, H. \& EcKhardt, B. 2004 A low dimensional model for turbulent shear flows. New J. Phys. 6, Article 56.

Moenlis, J., Faisst, H. \& Eckhardt, B. 2005 Periodic orbits and chaotic sets in a low-dimensional model for shear flows. SIAM J. Appl. Dyn. Syst. 4, 352-376.

Moenlis, J., Smith, T. R., Holmes, P. \& Faisst, H. 2002 Models for turbulent plane Couette flow using the proper orthogonal decomposition. Phys. Fluids 14, 2493-2507.

Moffatt, H. K. 1990 Fixed points of turbulent dynamical systems and suppression of nonlinearity. In Whither Turbulence? Turbulence at the Crossroads (ed. J. L. Lumley), pp. 250-257. Springer.

NAGATA, M. 1990 Three-dimensional finite-amplitude solutions in plane Couette flow: bifurcation from infinity. J. Fluid Mech. 217, 519-527.

PeYret, R. 2002 Spectral Methods for Incompressible Viscous Flow. Springer.

Podvin, B. 2001 On the adequacy of the ten-dimensional model for the wall layer. Phys. Fluids 13, 210-224.

Podvin, B. \& Lumley, J. 1998 A low-dimensional approach for the minimal flow unit. J. Fluid Mech. 362, 121-155.

Pope, S. 2000 Turbulent Flows. Cambridge University Press.

Reddy, S. C., Schmid, P. J., Baggetr, J. S. \& Henningson, D. S. 1998 On stability of streamwise streaks and transition thresholds in plane channel flows. J. Fluid Mech. 365, 269-303.

SANGHI, S. \& AubRY, N. 1993 Mode interaction models for near-wall turbulence. J. Fluid Mech. 247, 455-488.

Schmid, P. J. \& Henningson, D. S. 2000 Stability and Transition in Shear Flows. Springer.

Schmiegel, A. 1999 Transition to turbulence in linearly stable shear flows. PhD thesis, Universität Marburg.

Schmiegel, A. \& Eckhardt, B. 1997 Fractal stability border in plane Couette flow. Phys. Rev. Lett. 79, 5250-5253.

Sirovich, L. 1987 Turbulence and the dynamics of coherent structures, parts I-III. Q. Appl. Maths XLV (3), 561-582.

Sirovich, L. \& Zhou, X. 1994a Dynamical model of wall-bounded turbulence. Phys. Rev. Lett. 72 , 340-344.

Sirovich, L. \& Zhou, X. $1994 b$ Reply to 'Observations regarding 'Coherence and chaos in a model of a turbulent boundary layer' by X. Zhou and L Sirovich'. Phys. Fluids 6, 1579-1582. 
Smith, T. R. 2003 Low-dimensional models of plane Couette flow using the proper orthogonal decomposition. PhD thesis, Princeton University.

Smith, T. R., Moehlis, J. \& Holmes, P. 2004 Low-dimensional modelling of turbulence using the proper orthogonal decomposition: a tutorial. Nonlinear Dyn. (in press).

Smith, T. R., Moenlis, J. \& Holmes, P. 2005 Heteroclinic cycles and periodic orbits for the $\mathrm{O}(2)$-equivariant 0:1:2 mode interaction. Physica $D$ (submitted).

Smith, T. R., Moehlis, J. \& Holmes, P. J. 2003 Modeling and control of minimal flow unit turbulence in plane Couette flow. In Proc. IEEE Conference on Decision and Control, pp. 2322-2327. IEEE.

SQuire, H. B. 1933 On the stability for three-dimensional disturbances of viscous fluid flow between parallel walls. Proc. R. Soc. Lond. A 142, 621-628.

Trefethen, L. N. 1992 Pseudospectra of matrices. In Numerical Analysis 1991 (ed. D. F. Griffiths \& G. A. Watson), pp. 234-266. Longman.

Trefethen, L. N., Trefethen, A. E., Reddy, S. \& Driscoll, T. 1993 Hydrodynamic stability without eigenvalues. Science 261, 578-584.

WALEFFE, F. 1995a Hydrodynamic stability and turbulence: beyond transients to a self-sustaining process. Stud. Appl. Maths 95, 319-343.

WALEFFE, F. $1995 b$ Transition in shear flows. Nonlinear normality versus non-normal linearity. Phys. Fluids 7, 3060-3066.

WalefFe, F. 1997 On a self-sustaining process in shear flows. Phys. Fluids 9, 883-900.

Webber, G. A., Handler, R. A. \& Sirovich, L. 1997 The Karhunen-Loéve decomposition of minimal channel flow. Phys. Fluids 9, 1054-1066.

ZANG, T. A. \& Hussaini, M. Y. 1985 Numerical experiments on subcritical transition mechanisms. AIAA Paper 85-0296, pp. 1-18.

Zhou, X. \& Sirovich, L. 1992 Coherence and chaos in a model of a turbulent boundary layer. Phys. Fluids A 46, 2855-2874. 